\section{Shape resonances and the excitation of helium autoionising states by electrons in the $57-66 \mathrm{eV}$ region}

To cite this article: P J M van der Burgt et al 1986 J. Phys. B: At. Mol. Phys. 192015

View the article online for updates and enhancements.
Related content - A new type of $\mathrm{He}^{-}$resonance states in the
$\mathrm{P} J \mathrm{M}$ van der Burgt and H G M Heideman
- Orbital angular momentum exchange in
post-collision interaction
$\mathrm{P} J \mathrm{M}$ van der Burgt, J van Eck and H G M Heideman
- A model for post-collision interaction
based on an optical-potential description W van de Water, H G M Heideman and G Nienhuis

\section{Recent citations}

$$
\begin{aligned}
& \text { - Positron attachment to the } \mathrm{He}\left(\mathrm{ns}^{\wedge}\{2\}\right. \\
& \frac{\left.\{1\} S^{\wedge}\{\mathrm{e}\}\right) \text { states }}{\text { J. Mitroy and J. Grineviciute }} \\
& \text { - Positron Attachment to the He Doubly } \\
& \frac{\text { Excited States }}{\text { M. Bromley et al }} \\
& \text { - The post-collision energy shift of the } \\
& \frac{\left(n p^{5}(n+1) s^{2}\right)^{2} P}{\text { spectra of sodium }(n=2) \text { and caesium }}(n \\
& \frac{\text { s) atoms excited by electron impact }}{\text { A A Borovik and G N Ogurtsov }}
\end{aligned}
$$




\title{
Shape resonances and the excitation of helium autoionising states by electrons in the $57-66 \mathrm{eV}$ region
}

\author{
P J $M$ van der Burgt, J van Eck and $H$ G M Heideman \\ Fysisch Laboratorium, Rijksuniversiteit Utrecht, Princetonplein 5, $3584 \mathrm{CC}$ Utrecht, The \\ Netherlands
}

Received 9 September 1985

\begin{abstract}
We present optical excitation functions of singly excited helium states, measured by detecting the yield of emitted photons as a function of the incident electron energy from 56 to $66 \mathrm{eV}$. Many structures are observed, which are caused by negative-ion resonances and by the decay of autoionising states followed by post-collision interaction. Some of the structures are interpreted as being caused by hitherto unknown shape resonances lying very close to the thresholds of a particular class of autoionising states. As these shape resonances almost exclusively decay to their respective parent (autoionising) states, thereby considerably enhancing the threshold excitation cross sections of these states, they can only be observed via the PCI effect on the excitation functions of (higher lying) singly excited states. Using the recently introduced supermultiplet classification for doubly excited states a selection rule for the near-threshold excitation of doubly excited states by electron impact is deduced from the measurements. Only states with large probabilities in the Wannier region of configuration space (where the two electrons are at nearly equal distances and on opposite sides of the nucleus) are strongly excited. It is pointed out that these states are precisely the states that can support the above mentioned shape resonances at their thresholds.
\end{abstract}

\section{Introduction}

During the last two decades much attention has been paid to the study of resonances in inelastic electron-helium scattering. On the incident energy scale two regions are of particular interest. The first region from $19-25 \mathrm{eV}$ covers the thresholds of singly excited helium states. The electron impact excitation of singly excited states in this energy range has been studied experimentally by detecting metastable helium atoms (Buckman et al 1983), inelastically scattered electrons (Andrick 1979, Phillips and Wong 1981 and references therein) and emitted photons (e.g. Heideman et al 1976, Heddle 1977). All these measurements showed numerous doubly excited $\mathrm{He}^{-}$resonances which significantly affect the inelastic excitation cross sections. The resonances have also been studied theoretically (e.g. Nesbet 1978, Watanabe 1982, Freitas et al 1984). Two classes of resonances are distinguished (Nesbet 1978, Freitas et al 1984): one class in which both excited electrons are in a highly correlated state, the other class formed by an electron very weakly bound in the polarisation potential of a singly excited state. The experiment of Buckman et al (1983) showed that the resonances occur in distinct groups; the energies of the lowest members of each group could be fitted to a modified Rydberg formula that takes into account the two-electron correlations in the doubly excited resonances (Read 1983).

The second region in electron-helium scattering from 57-66 eV covers the thresholds of doubly excited states. The two-electron correlations which play a primary role in 
these states became apparent for the first time in the measurements of Madden and Codling (1963) and have since then been the subject of numerous studies (Fano 1983, Lin 1984 and references therein). Contrary to the singly excited states the doubly excited states decay to the ground state of the $\mathrm{He}^{+}$ion by ejecting an electron. Doubly excited states of a particular class, the so-called parity unfavoured states such as $\mathrm{He}^{* *}\left(2 \mathrm{p}^{2}\right)^{3} \mathrm{P}$, are stable against autoionisation due to conservation of parity; these states decay by photoemission. The study of the near-threshold excitation of autoionising states is complicated by the so-called post-collision interaction (PCI), first reported by Hicks et al (1974). This effect causes an energy exchange between the scattered and the ejected electron (the ejected electron gains energy at the cost of the slow scattered electron), which increases with decreasing energy of the scattered electron. At incident energies close enough to the thresholds of autoionising states this energy exchange may result in the capture of the scattered electron into a singly excited state of the neutral atom (Heideman et al 1974, Smith et al 1974), thereby causing structures on the excitation curves of these states. As a result the excitation of autoionising states close to their thresholds can much better be studied via the excitation of singly excited states than via the detection of ejected (autoionisation) electrons. Further, since PCI most prominently affects the higher lying $(n>3)$ singly excited states, which cannot be separated when the inelastically scattered electrons are observed, the near-threshold excitation of autoionising states is most conveniently studied by our technique of detecting the photons emitted by the singly excited states following their excitation (see e.g. Heideman 1980).

Analogous to the threshold excitation of singly excited states in the $19-25 \mathrm{eV}$ range we expect that negative ion resonances significantly affect the excitation cross sections of autoionising states in the $57-66 \mathrm{eV}$ region. The question arises whether also in this region modified Rydberg series of resonances exist. The importance of resonances in the excitation of autoionising states of helium was already suggested by Nesbet (1976). In this paper we present optical excitation functions measured with a higher energy resolution of the incident electron beam as compared with previous experiments (van Ittersum et al 1976, Heideman 1980). A summary and overview of the results was given previously (van der Burgt and Heideman 1985).

\section{Mechanisms for indirect excitation of singly excited states}

Before attempting an analysis of the structures seen in the excitation functions we shall first focus our attention on the various mechanisms for indirect excitation of singly excited helium states in the $57-66 \mathrm{eV}$ region. These mechanisms involve negative ion resonances and the post-collision interaction following the excitation of autoionising states. In the excitation of a helium atom by an electron three reaction mechanisms may result in a singly excited state and a free electron:

$$
\begin{aligned}
& \mathrm{e}+\mathrm{He} \rightarrow \mathrm{He}^{-} \longrightarrow \mathrm{He}^{*}+\mathrm{e}_{\mathrm{ej}}
\end{aligned}
$$

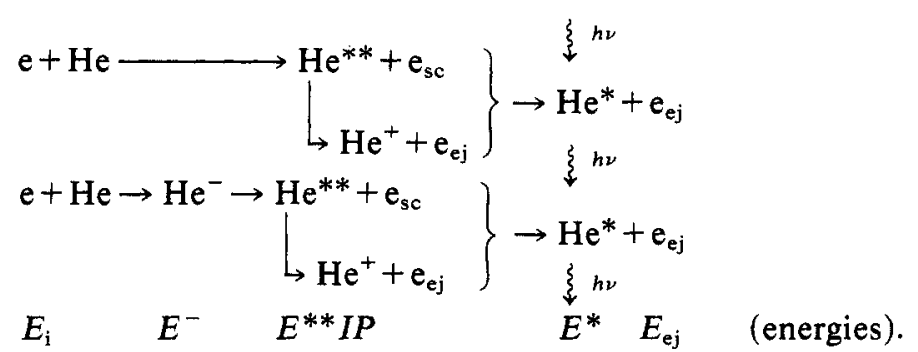


Structures due to these mechanisms are observed on an interfering background due to direct excitation of the singly excited state involved:

$$
\mathrm{e}+\mathrm{He} \longrightarrow \mathrm{He}^{*}+\mathrm{e}_{\mathrm{ej}} .
$$

\subsection{Negative ion resonance}

According to the first reaction mechanism (i) a negative ion resonance decays directly to a singly excited state and a Beutler-Fano resonance profile is observed in the optical excitation functions in a narrow range around a fixed position $E_{\mathrm{i}}=E^{-}$. The resonant state decays when one of the electrons makes a transition to the $1 \mathrm{~s}$ orbital and another electron is ejected. This reaction mechanism is mainly responsible for decay of resonances of the intrashell type where all three electrons in the $\mathrm{He}^{-}$state are in the $n=2$ shell.

\subsection{Autoionisation and post-collision interaction}

Autoionising states decay by electron ejection and can only be observed in excitation functions when the scattered electron loses so much energy during PCI that it is captured into a singly excited state. This is illustrated in figure 1 . In the absence of $\mathrm{PCI}$, at incident energies much higher than the energy of the autoionising state, a Beutler-Fano resonance profile is observed at the position $E_{\mathrm{ej}}=E^{* *}-I P$ in the yield of ejected electrons due to interference with the direct ionisation of helium (that is, when the ejected electron yield is measured as a function of the ejected electron energy at a fixed value of the incident electron energy). During the post-collision interaction energy is exchanged between both electrons: $E_{\mathrm{sc}}<E_{\mathrm{i}}-E^{* *}$ and $E_{\mathrm{ej}}>E^{* *}-I P$.

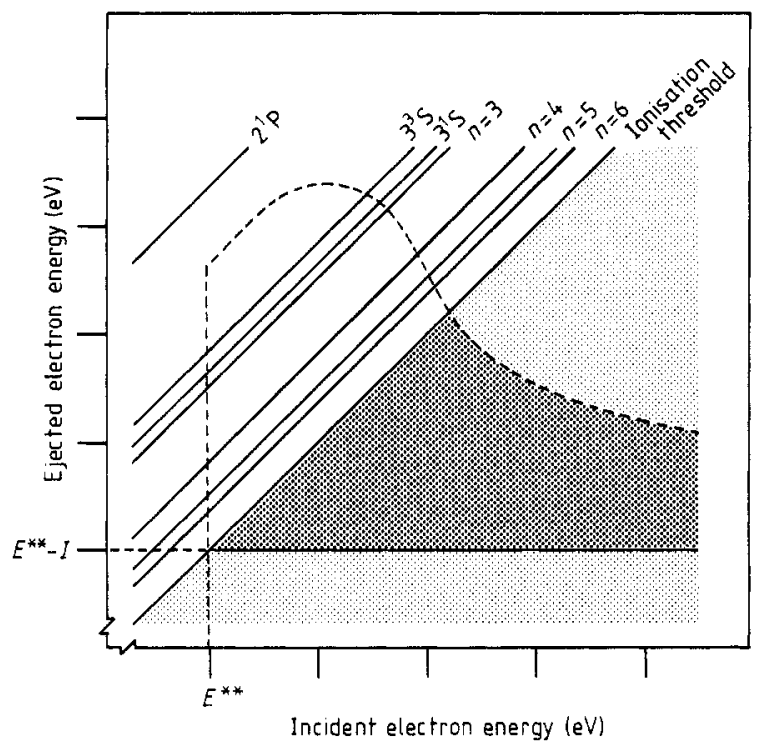

Figure 1. Energy distribution of ejected electrons after the post-collision interaction. The straight lines at constant energy loss $E_{l}=E_{\mathrm{i}}-E_{\mathrm{ej}}$ correspond to the capture of the scattered electron into a singly excited state of the helium atom. It is assumed that PCI excitation of $n=2$ states is not possible. 
Observation of PCI in ejected electron spectra is complicated by interference with the direct ionisation background (see Baxter et al 1979a, van der Burgt et al 1985a). Due to interference an oscillatory PCI profile is observed rather than a PCI broadened peak.

Capture of the scattered electron into a singly excited state with energy $E^{*}$ takes place when the energy exchange results in $E_{\mathrm{sc}}<0$ and $E_{\mathrm{ej}}=E_{\mathrm{i}}-E^{*}>E_{\mathrm{i}}-I P$. This is illustrated in figure 1 where singly excited states appear as straight sloping lines $E_{\mathrm{ej}}=E_{\mathrm{i}}-E^{*}$ above the ionisation threshold $E_{\mathrm{ej}}=E_{\mathrm{i}}-I P$. When the incident energy is lowered towards the threshold of the autoionising state the probability for excitation of lower $n$ states becomes larger because the residual energy $E_{\mathrm{i}}-E^{* *}$ of the scattered electron before PCI is smaller, and consequently the average exchange of energy during PCI is larger. In addition, because there must be a maximum of the energy exchanged during $\mathrm{PCI}$, no $\mathrm{PCI}$ excitation is possible of singly excited states which lie too far below the ionisation threshold. Again interference between PCI excitation and direct excitation of singly excited states determines the appearance of structures in the excitation functions. The phase of the PCI amplitude varies strongly with the excess energy above the threshold of the autoionising states, and thus with the incident electron energy, but also with the principal quantum number $n$ of the singly excited states (see $\$ 4$ ). As a result the PCI structures appear as asymmetrical oscillatory profiles which seem to shift to higher energies with the increase of the principal quantum number $n$. This energy shift distinguishes $\mathrm{PCr}$ structures due to mechanism (ii) from resonance structures due to mechanism (i).

\subsection{Excitation of an autoionising state via a negative ion resonance}

The third mechanism (iii) for excitation of singly excited states is related to the second and involves excitation of an autoionising state via a negative ion resonance.

Uptil now only two negative ion resonances in the $57-66 \mathrm{eV}$ region have been identified unambiguously in the measurements. They are the $\mathrm{He}^{-}\left(2 \mathrm{~s}^{2} 2 \mathrm{p}\right)^{2} \mathrm{P}$ resonance at $57.22 \mathrm{eV}$ and the $\mathrm{He}\left(2 \mathrm{~s} 2 \mathrm{p}^{2}\right)^{2} \mathrm{D}$ resonance at $58.30 \mathrm{eV}$. These resonances are of the intrashell type, formed by three electrons in the $n=2$ shell at approximately equal distances from the nucleus. Fano and Cooper (1965) suggest that the relevant configurations of $\mathrm{He}^{-}$have all three electrons in the $n=2$ shell. Only four resonances of this type are accessible, with configurations $\mathrm{He}^{-}\left(2 \mathrm{~s}^{2} 2 \mathrm{p}\right)^{2} \mathrm{P}, \mathrm{He}^{-}\left(2 \mathrm{~s} 2 \mathrm{p}^{2}\right)^{2} \mathrm{D}, \mathrm{He}^{-}\left(2 \mathrm{~s} 2 \mathrm{p}^{2}\right)^{2} \mathrm{~S}$ and $\mathrm{He}^{-}\left(2 \mathrm{p}^{3}\right)^{2} \mathrm{P}$.

Nesbet (1978) and Freitas et al (1984) have proposed that in the region of the singly excited $n=3$ thresholds two classes of resonances exist. Resonances of the first class are formed by two excited electrons in the $n=3$ shell moving in a highly correlated state, analogous to the intrashell doubly excited states of $\mathrm{H}^{-}$and $\mathrm{He}$. Resonances of the second class are closely associated with a single threshold, and are presumably formed by an electron at a large distance, very weakly bound in the polarisation potential of the parent state. These intershell type resonances almost exclusively decay to their parent states. It appears that the polarisation potential is not always strong enough to bind a third electron so that instead a virtual state may occur (Nesbet 1978). In a previous paper (van der Burgt and Heideman 1985) we have used the terminology of Nesbet (1978), who refers to the two classes of resonances as valence shell and non-valence resonances, respectively.

In analogy with the resonances near the $n=3$ thresholds it may well be that intershell resonances or virtual states exist also near the thresholds of the doubly excited states. These resonances, in which one electron at a relatively large distance is weakly bound 
in the polarisation potential of a doubly excited state, are expected to occur close to the thresholds of the doubly excited states. As the intershell resonances are expected to decay almost exclusively to their parent autoionising states, these resonances will probably give rise to observable structures only in the higher $n$ singly excited states via the post-collision interaction (mechanism (iii)). They will not occur in the excitation cross sections of the $n=2$ singly excited states, which are not, or very weakly, excited via the PCI mechanism. On the other hand the $\mathrm{He}^{-}\left(2 \mathrm{~s}^{2} 2 \mathrm{p}\right)^{2} \mathrm{P}$ and $\mathrm{He}^{-}\left(2 \mathrm{~s} 2 \mathrm{p}^{2}\right)^{2} \mathrm{D}$ intrashell resonances are expected to decay mainly to the lower $n$ singly excited states directly (mechanism (i)) and hence to occur predominantly in the excitation cross sections of the $n=2$ singly excited states (see figure 9 and Roy et al 1978b). The $\mathrm{He}^{-}\left(2 \mathrm{~s} 2 \mathrm{p}^{2}\right)^{2} \mathrm{D}$ resonance also enhances the excitation cross section of the $\mathrm{He}^{* *}\left(2 \mathrm{~s}^{2}\right)^{1} \mathrm{~S}$ autoionising state at $0.48 \mathrm{eV}$ above its threshold (see van der Burgt et al 1985a). Thus it is to be expected that the pattern of resonance structures in the excitation of the low lying $(n=2)$ states differs appreciably from that in the excitation of the higher lying states, the former being exclusively caused by the intrashell resonances, the latter also by the intershell resonances via the PCI mechanism.

\subsection{Classification of autoionising states}

The existence of an intershell resonance near an autoionisation threshold is obviously related to the polarisability $\dagger$ of the autoionising state and thus to the correlated state of the two electrons in the doubly excited atom. In order to gain some insight in such a relationship it is sensible to use a classification scheme for doubly excited states that is based upon the analysis of electron correlations. Very recently such a classification scheme was proposed by Lin $(1983,1984)$. This classification scheme is unique for all states of a two-electron atom and is based upon the study of two-electron correlations in hyperspherical coordinates (Lin 1974, Fano 1983) and the supermultiplet classification of intrashell doubly excited states (Herrick and Kellman 1980, Herrick et al 1980). The quantum numbers $(K, T)^{A}$ are introduced where $K$ and $T$ (Sinanoglu and Herrick 1975) replace the orbital angular momentum quantum numbers $l_{1}$ and $l_{2}$ of both electrons and are used to describe the angular correlations between the two electrons. The quantum number $A$ is introduced (Lin 1984) to distinguish different types of radial correlations. The classification is denoted as

$$
{ }_{n}(K, T)_{N}^{A}{ }^{2 S+1} L^{\pi}
$$

where $n$ is the principal quantum number of the outer electron and $N$ that of the inner electron: $N$ thus indicates the $\mathrm{He}^{+}$dissociation limit if $n \rightarrow \infty$. States with identical $(K, T)^{A}, A= \pm 1$, but different $L, S$ and $\pi$ (orbital angular momentum, spin and parity, respectively) have isomorphic correlation patterns (Lin 1984) and exhibit a multiplet structure which may be interpreted in terms of a collective rotational and bending vibrational motion, analogous to the rovibrational motion of linear triatomic molecules (Kellman and Herrick 1980). We have given the classification of Lin (1984) in table 1 for a number of $\pi=(-1)^{L}$ states of $\mathrm{He}^{* *}$.

\footnotetext{
+ We use the word polarisation in a general sense, indicating the perturbed motion of the atomic electrons in the presence of a near scattered electron, contrary to the more explicit indication of a $-1 / r^{4}$ potential, often used.
} 
Table 1. A number of autoionising states of $\mathrm{He}$ in the $N=2$ channels. Only doubly excited states of parity $\pi=(-1)^{L}$, which can decay by autoionisation, are included in the table. Above $63 \mathrm{eV}$ the table is incomplete.

\begin{tabular}{lcll}
\hline$\left(N l, n l^{\prime}\right)^{1,3} L$ & ${ }_{n}(K, T)_{N}^{A}{ }^{1,3} L^{\pi}$ & Energy $(\mathrm{eV})$ & Width $(\mathrm{eV})$ \\
\hline $\mathrm{He}^{* *}\left(2 \mathrm{~s}^{2}\right)^{1} \mathrm{~S}$ & $\mathrm{He}^{* *}{ }_{2}(1,0)_{2}^{+1} \mathrm{~S}^{\mathrm{e}}$ & $57.82^{\mathrm{a}}$ & $0.138^{\mathrm{a}}$ \\
$(2 \mathrm{~s} 2 \mathrm{p})^{3} \mathrm{P}$ & ${ }_{2}(1,0)_{2}^{+3} \mathrm{P}^{\mathrm{o}}$ & $58.30^{\mathrm{a}}$ & $0.008^{\mathrm{d}}$ \\
$\left(2 \mathrm{p}^{2}\right)^{1} \mathrm{D}$ & ${ }_{2}(1,0)_{2}^{+1} \mathrm{D}^{\mathrm{e}}$ & $59.90^{\mathrm{a}}$ & $0.072^{\mathrm{a}}$ \\
$(2 \mathrm{~s} 2 \mathrm{p})^{1} \mathrm{P}$ & ${ }_{2}(0,1)_{2}^{+1} \mathrm{P}^{\mathrm{o}}$ & $60.130^{\mathrm{b}}$ & $0.038^{\mathrm{b}}$ \\
$\left(2 \mathrm{p}^{2}\right)^{1} \mathrm{~S}$ & ${ }_{2}(-1,0)_{2}^{+1} \mathrm{~S}^{\mathrm{e}}$ & $62.06^{\mathrm{a}}$ & \\
$(2 \mathrm{~s} 3 \mathrm{~s})^{3} \mathrm{~S}$ & ${ }_{3}(1,0)_{2}^{-3} \mathrm{~S}^{\mathrm{e}}$ & $62.62^{\mathrm{c}}$ & \\
$(23 \mathrm{sp}-)^{1} \mathrm{P}$ & ${ }_{3}(1,0)_{2}^{-1} \mathrm{P}^{\mathrm{o}}$ & $62.758^{\mathrm{b}}$ & \\
$(2 \mathrm{~s} 3 \mathrm{~s})^{1} \mathrm{~S}$ & ${ }_{3}(1,0)_{2}^{+1} \mathrm{~S}^{\mathrm{e}}$ & $62.94^{\mathrm{a}}$ & $0.041^{\mathrm{a}}$ \\
$(23 \mathrm{sp}+)^{3} \mathrm{P}$ & ${ }_{3}(1,0)_{2}^{+3} \mathrm{P}^{\mathrm{o}}$ & $63.07^{\mathrm{a}}$ & \\
$(2 \mathrm{p} 3 \mathrm{p})^{1} \mathrm{D}$ & ${ }_{3}(1,0)_{2}^{+1} \mathrm{D}^{\mathrm{e}}$ & $63.50^{\mathrm{a}}$ & \\
$(2 \mathrm{~s} 4 \mathrm{~s})^{1} \mathrm{~S}$ & ${ }_{4}(1,0)_{2}^{+1} \mathrm{~S}^{\mathrm{e}}$ & $64.18^{\mathrm{a}}$ & \\
$(24 \mathrm{sp}+)^{3} \mathrm{P}$ & ${ }_{4}(1,0)_{2}^{+3} \mathrm{P}^{\mathrm{o}}$ & $64.23^{\mathrm{a}}$ & \\
$(2 \mathrm{p} 4 \mathrm{p})^{1} \mathrm{D}$ & ${ }_{4}(1,0)_{2}^{+1} \mathrm{D}^{\mathrm{e}}$ & $64.39^{\mathrm{a}}$ & \\
\hline
\end{tabular}

${ }^{a}$ Hicks and Comer (1975), ${ }^{b}$ Madden and Codling (1965), ${ }^{c}$ Lipsky et al (1977) (calculated value), ${ }^{d}$ Cederquist et al (1983).

\section{Experiment}

\subsection{Detection of photons}

The apparatus used for the present study consists of a conventional electron monochromator combined with a photon detection system. Electrons emitted by a tungsten filament are focused on the entrance of a hemispherical energy selector by cylindrical electrostatic lenses. After passing the energy selector, which is operated at a resolution of about $110 \mathrm{meV}$, the beam is directed into the interaction chamber containing the helium gas at a pressure of 0.02 Torr. The analyser part of the spectrometer, used in other experiments for the detection of scattered electrons, is set at $0^{\circ}$ and is used as a Faraday cup for the incident electron beam. For this purpose the first two diaphragms of 0.6 and $1.5 \mathrm{~mm}$ diameter, respectively, in the analyser part were replaced by $3 \mathrm{~mm}$ diameter ones.

In order to detect photons emitted in a large solid angle the interaction chamber was equipped with a gold coated spherical mirror at the bottom and an optical fibre at the top. The complete optical set up of the apparatus is sketched in figure 2.

The spherical mirror has a radius such that a section of $5 \mathrm{~mm}$ length of the incident beam is focused with a magnification factor equal to 2 on the front side of the optical fibre which has a diameter of $10 \mathrm{~mm}$. Thus photons emitted within a cone with a top angle of about $115^{\circ}$ are reflected into the optical fibre. Photons may also enter the fibre directly, but this is only a small fraction compared with the number of photons reflected by the mirror. Photons of the required wavelength are detected using an interference filter and a photomultiplier. For photons divergent from normal incidence the central wavelength $\lambda_{0}$ transmitted by an interference filter shifts to a lower 


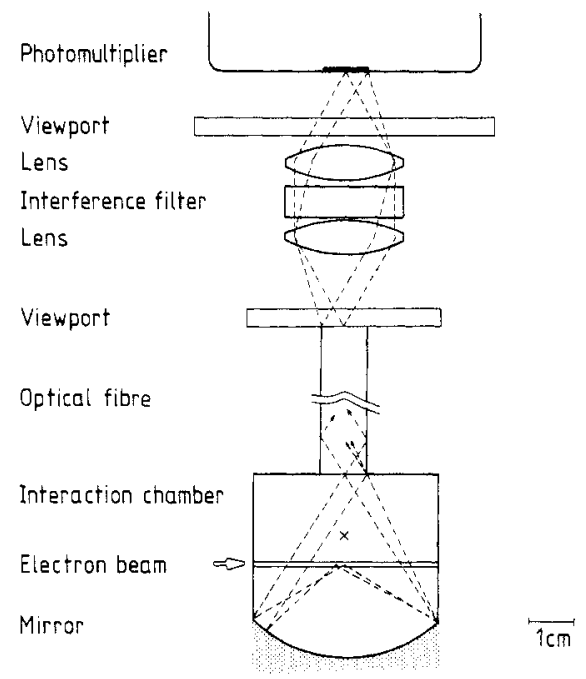

Figure 2. Experimental set-up for the detection of photons.

wavelength $\lambda_{\alpha}$ according to the formula

$$
\lambda_{\alpha}=\lambda_{0}\left[1-\left(n_{\mathrm{e}}^{2} / n^{2}\right) \sin ^{2} \alpha\right]^{1 / 2}
$$

where $\alpha$ is the angle of incidence of the photon and $n$ and $n_{\mathrm{e}}$ are the indices of refraction of the filter and the external medium (air), respectively. Therefore two glass lenses are installed, one before and one behind the interference filter, to reduce the maximum angle of incidence to $14^{\circ}$. In four cases $(396.5 \mathrm{~nm}, 416.9 \mathrm{~nm}, 443.8 \mathrm{~nm}$, $447.2 \mathrm{~nm}$ ) adjacent transitions are so close that this angle has to be reduced even further by enlarging the distance between the fibre exit and the photomultiplier and by using lenses with a greater focal length. When measuring uv transitions $(294.5 \mathrm{~nm}$, $318.8 \mathrm{~nm}$ ) the glass lenses and viewport are replaced by fused silica ones. The EMI 9863 QB/350 photomultiplier used is sensitive for wavelengths in the $200-700 \mathrm{~nm}$ range with a maximum near $400 \mathrm{~nm}$. A somewhat different set-up for the detection of photons which includes a mirror is used by Leisin et al (1985).

The pulses from the photomultiplier are stored and accumulated in a multichannel analyser, the advance address of which is swept synchronously with the incident electron energy. The multichannel analyser is connected to a data storage and communication system (de Raaf 1983) facilitating transport of the multichannel data to a PDP 11/70 computer for further off-line analysis. The present experimental equipment has several advantages compared with the equipment used previously at our laboratory (see van Ittersum et al 1976): the energy resolution of the incident beam is about $110 \mathrm{meV}$ ( $250 \mathrm{meV}$ previously), photons emitted in a large solid angle are detected and the interference filters have a high transmission compared with a monochromator. As the central wavelength of an interference filter shifts with angle of incidence, filters can be used only for transitions which lie not too close to neighbouring transitions. Therefore in practice only $n=3,4$ and 5 (and occasionally 6) excitation functions can be measured, with the exclusion of the $4^{1} \mathrm{~S}-2^{1} \mathrm{P}(504.8 \mathrm{~nm})$ transition which lies close to the very strong $3^{1} \mathrm{P}-2^{1} \mathrm{~S}(501.6 \mathrm{~nm})$ transition, and the $5^{3} \mathrm{D}-2^{3} \mathrm{P}(402.6 \mathrm{~nm})$ transition which almost coincides with the $7^{1} \mathrm{~S}-2^{1} \mathrm{P}(402.4 \mathrm{~nm})$ transition. The electron spectrometer could have been operated at a resolution better than $110 \mathrm{meV}$, were it not 
that the beam current would then become too small to reach acceptable statistics. In the present situation measuring times were typically one to three days per spectrum, in two cases even five days.

\subsection{Experimental results}

We have measured seven series of excitation functions, presented in figures 3-9. Figures 3-8 show the optical excitation functions of the higher lying $(n \geqslant 3)$ states,

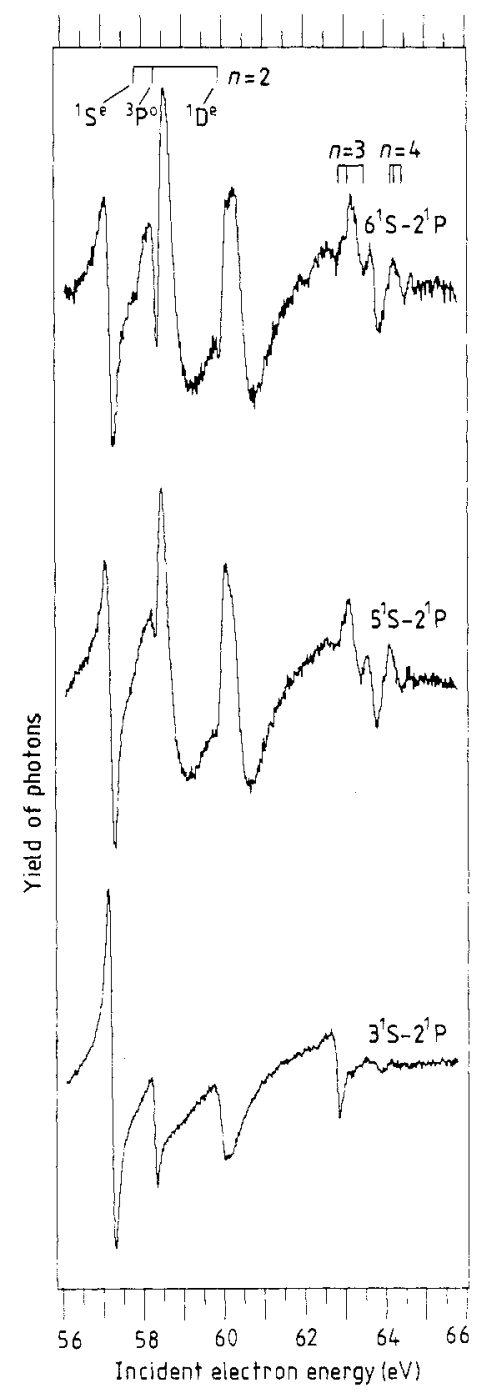

Figure 3. The excitation of the $n^{1} \mathrm{~S}$ states of helium as a function of the incident electron energy. Subsequent groups of ${ }^{1} \mathrm{~S}^{e},{ }^{3} \mathrm{P}^{\circ}$ and ${ }^{1} \mathrm{D}^{\mathrm{e}}$ autoionising states give rise to groups of $\mathrm{PCI}$ profiles in the excitation functions. We have indicated the thresholds of the autoionising states in the first three groups with the principal quantum number $n=2,3$ and 4 of the outer electron.

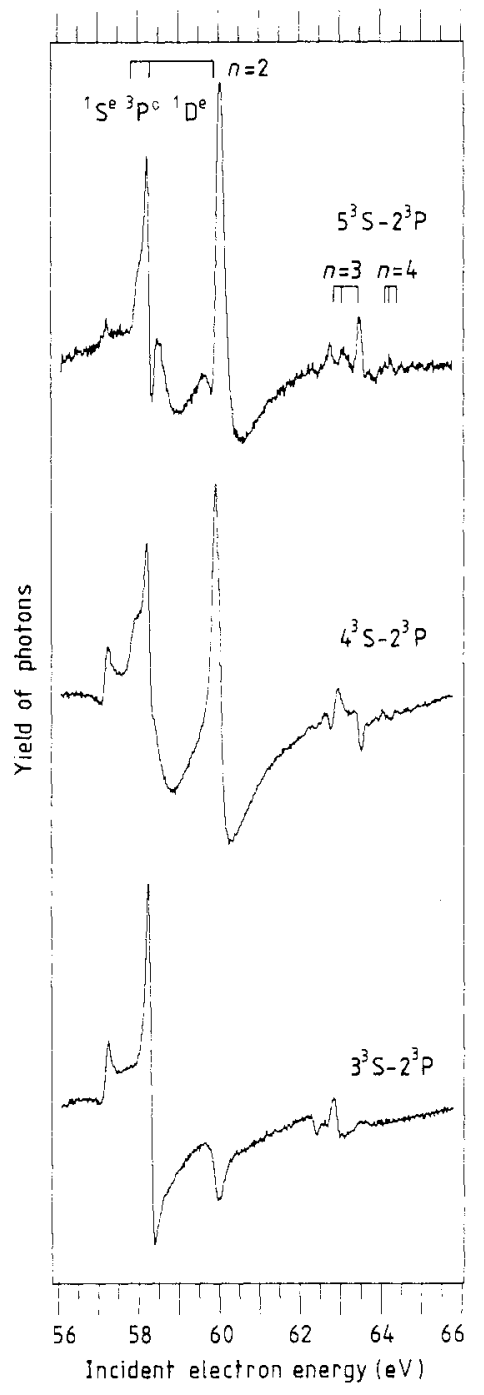

Figure 4. The excitation of the $n^{3} S$ states of helium as a function of the incident electron energy. Note the shifting PCI structure around $60 \mathrm{eV}$. The narrow peak observed at $59.90 \mathrm{eV}$ shows a very strong threshold excitation of the $\mathrm{He}^{* *}\left(2 \mathrm{p}^{2}\right)^{1} \mathrm{D}$ autoionising state caused by the presence of a negative ion resonance. 


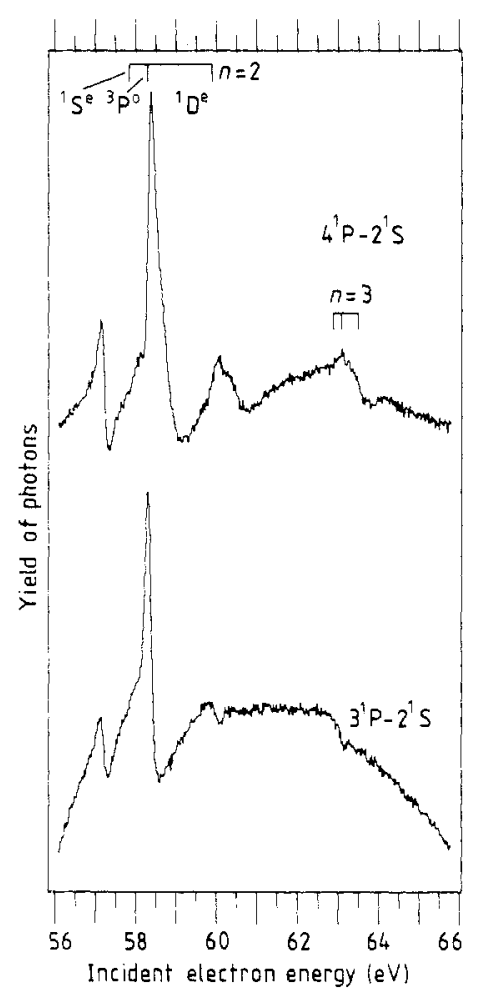

Figure 5. The excitation of the $n^{1} \mathrm{P}$ states of helium as a function of the incident electron energy. The curved background is due to the very high direct excitation of the $n^{1} \mathrm{P}$ states.

whereas figure 9 shows the differential excitation functions at $10^{\circ}$ scattering angle of the $2^{1} \mathrm{~S}$ and $2^{3} \mathrm{~S}$ states. The spectra in figure 9 were measured by detection of scattered electrons, the electron spectrometer being operated in the constant energy loss mode (see van der Burgt et al 1985a). As anticipated at the end of $\S 2.3$ the pattern of the resonance structures is much richer in the excitation of the $n \geqslant 3$ states than in the excitation of the $n=2$ states. A more detailed discussion of these results will be given in $\S 5$.

The measurements were performed at a gas pressure of 0.02 Torr. This pressure is too high for absolute excitation cross section measurements as imprisonment of resonance radiation and collisional transfer of excitation energy (Massey and Burhop 1969) may have some effect. We assume that these secondary processes have no disturbing effect on the $\mathrm{PCI}$ and resonance structures in the optical excitation functions. At a pressure of 0.02 Torr the mean free path length of the electrons is still much larger than the diameter of the gas cell.

The structures are influenced by cascading from higher lying states. Cascading is discussed to some extent by Defrance (1980). In estimating contributions due to cascading we have used cross sections measured by van Raan et al (1974), Scott and McDowell $(1975,1976)$ and lifetimes measured by Bukow et al (1977). Non-available cross sections may be estimated using the $n^{-3}$ scaling rule (Massey and Burhop 1969). We have found that only in the ${ }^{3} \mathrm{P}$ spectra, figure 6 , are there are significant cascade contributions to the observed structures. In the $3^{3} \mathrm{P}$ spectrum approximately $50 \%$ of the structure at $60.0 \mathrm{eV}$ is due to cascading fro.n the $4^{3} \mathrm{~S}$ level and $25 \%$ of the structure 


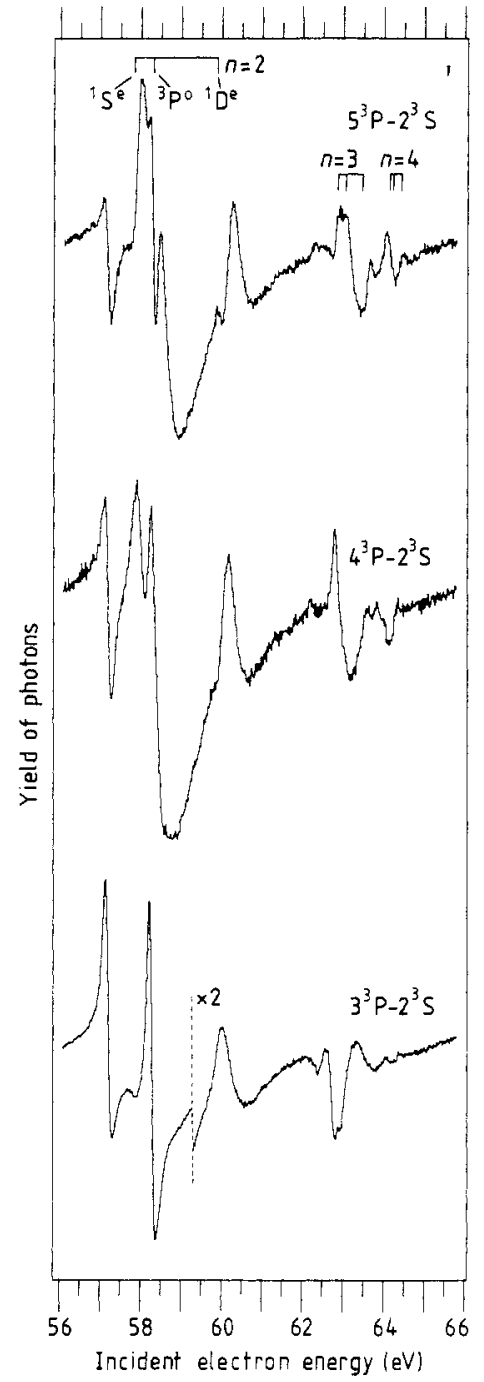

Figure 6. The excitation of the $n^{3} \mathrm{P}$ states of helium as a function of the incident electron energy. The broad and deep dip observed around $59 \mathrm{eV}$ is caused by the superposition of $\mathrm{PCI}$ profiles due to the $\mathrm{He}^{* *}\left(2 \mathrm{~s}^{2}\right)^{1} \mathrm{~S}$ and $\mathrm{He}^{* *}(2 \mathrm{~s} 2 \mathrm{p})^{3} \mathrm{P}$ autoionising states. The peak at $60 \mathrm{eV}$ in the $3^{3} \mathrm{P}$ excitation function is caused by cascading from the $n^{3} S$ levels.

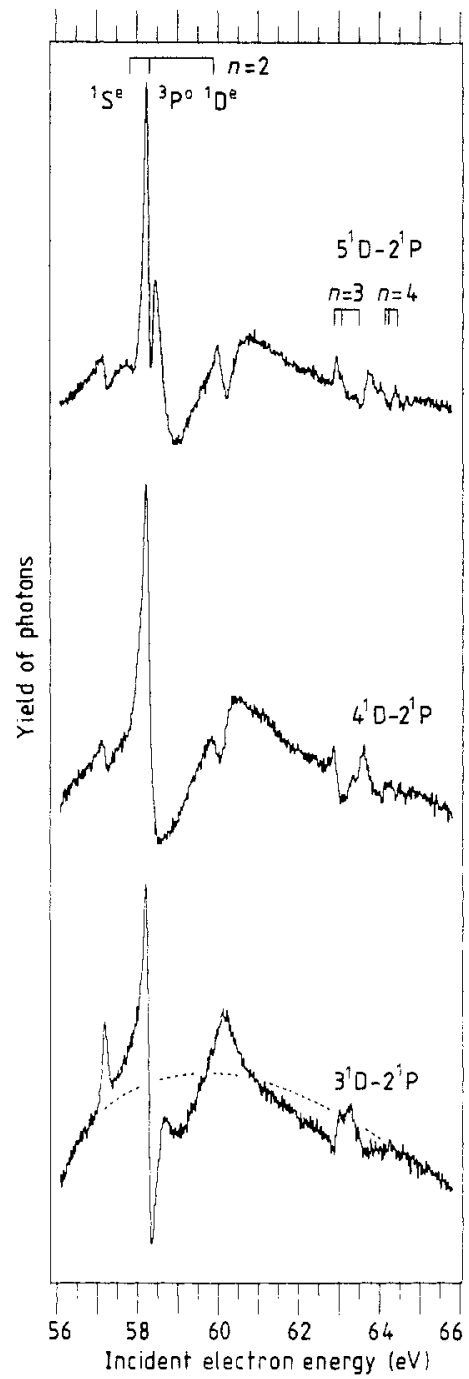

Figure 7. The excitation of the $n^{1} \mathrm{D}$ states of helium as a function of the incident electron energy. The narrow peak observed at $58.30 \mathrm{eV}$ in the $4^{1} \mathrm{D}$ excitation function shows a very strong threshold excitation of the $\mathrm{He}^{* *}(2 \mathrm{~s} 2 \mathrm{p})^{3} \mathrm{P}$ autoionising state caused by the presence of a negative ion resonance: compare with figure 5. The $60 \mathrm{eV}$ structures are interpreted as $\mathrm{PCl}$ structures: compare with the calculated profiles in figure $11(c)$.

at $58.3 \mathrm{eV}$ is due to cascading from the $4^{3} \mathrm{D}$ level. In the $4^{3} \mathrm{P}$ spectrum $10 \%$ of the structure at $60.0 \mathrm{eV}$ is due to cascading from the $5^{3} \mathrm{~S}$ level.

Incident energy scales were calibrated against the position of the $\mathrm{He}^{-}\left(2 \mathrm{~s}^{2} 2 \mathrm{p}\right)^{2} \mathrm{P}$ resonance at $57.22 \mathrm{eV}$ which lies below the thresholds of all autoionising states. As the range across which the incident energy is swept amounts to $10 \mathrm{eV}$, the incident energy scale may have shifted by some tens of $\mathrm{meV}$ above $63 \mathrm{eV}$, due to a change in space charge in the beam. From most of the spectra a linear sloping background was 


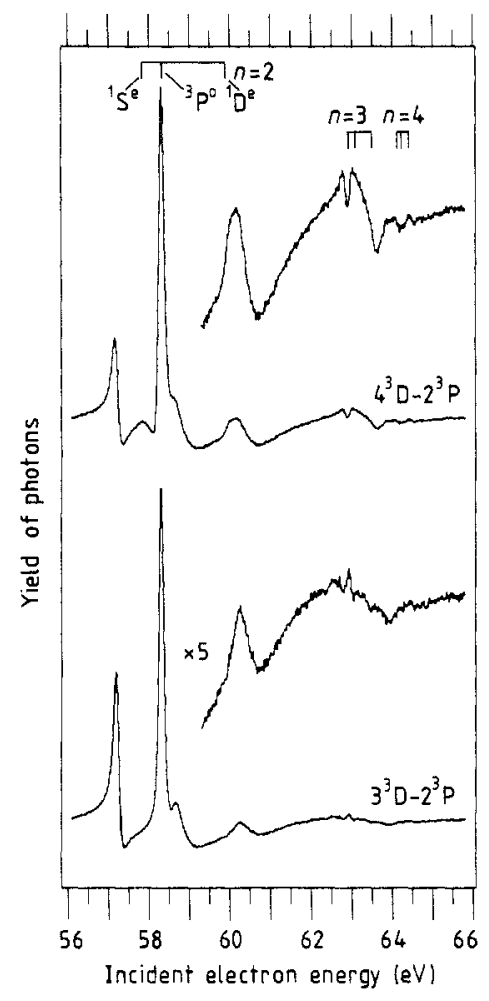

Figure 8. The excitation of the $n^{3} \mathrm{D}$ states of helium as a function of the incident electron energy. The heights of the peaks at $58.30 \mathrm{eV}$ are about $30 \%$ of the direct excitation cross section.

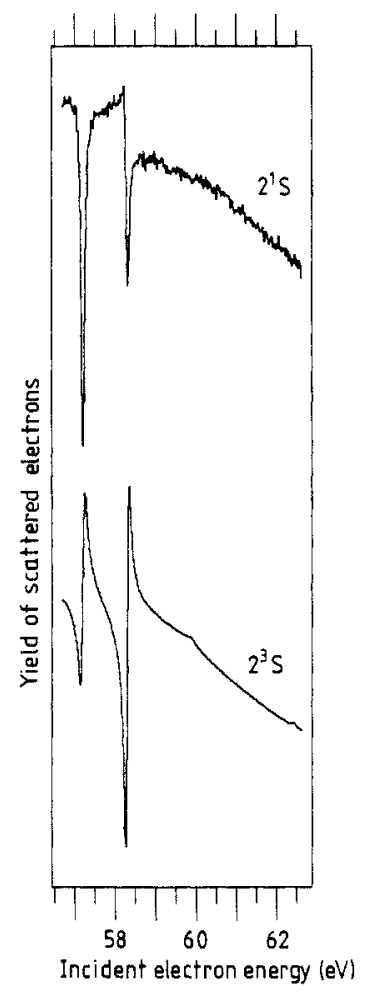

Figure 9. Yield of scattered electrons from the $2^{1} \mathrm{~S}$ and $2^{3} \mathrm{~S}$ states of helium. These measurements were performed in the constant energy loss mode of the electron spectrometer (see van de Burgt 1985a).

subtracted. The curved background in some of the spectra is due to a variation of the cross section for direct excitation of the singly excited state or due to a small variation of the incident beam current as a function of the incident electron energy.

\section{A model for post-collision interaction}

In addition to the discussion in $\$ 2.2$ a more detailed study of the post-collision interaction is fruitful for the general understanding of the PCI profiles observed in the excitation functions. A quantum mechanical model for PCI for the case that the scattered electron is captured into a singly excited state was formulated by Nienhuis and Heideman (1976) and applied to some practical cases by van de Water et al (1981). This model is based on a formal separation of PCI from the initial excitation and subsequent decay of the autoionising state. Similar to the resonance theory of Feshbach (1962) a standard optical potential technique is applied, which allows a separation of the transition amplitude into two parts, $T=T_{\mathrm{p}}+T_{\mathrm{opt}}, T_{\mathrm{p}}$ describing the direct excitation of singly excited states, and $T_{\mathrm{opt}}$ containing the effect of autoionising states on the excitation process. The transition amplitude becomes (Nienhuis and Heideman 1976, 
equation (3.10)):

$$
T_{\text {opt }}=\int \mathrm{d} \boldsymbol{k}_{\beta}\left\langle\boldsymbol{k}_{\alpha} f^{P-}|P H Q| \boldsymbol{k}_{\beta} a^{Q-}\right\rangle \frac{1}{E_{\mathrm{i}}-E^{* *}-\frac{1}{2} k_{\beta}^{2}+\frac{1}{2} \mathrm{i} \Gamma}\left\langle\boldsymbol{k}_{\beta} a^{Q-}|Q H P| \boldsymbol{k}_{\mathrm{i}} 0^{P+}\right\rangle
$$

where $|0\rangle$ denotes the ground state of the helium atom, $|a\rangle$ a doubly excited autoionising state, $|f\rangle$ the final singly excited state, and $|\boldsymbol{k}\rangle$ a free electron state of momentum $\boldsymbol{k}$. The indices $\alpha$ and $\beta$ indicate the ejected and scattered electron respectively. The projection operator $P$ projects on the subspace of states with at least one electron in a 1s orbital, the complementary operator $Q=I-P$ projects on the subspace containing the doubly excited states.

We assume that the decay of the autoionising state is not influenced by the scattered electron by replacing $P H Q$ in (4.1) by $P H_{\beta} Q$, where $H=H_{\beta}+V_{\beta}$ and $H_{\beta}$ is the free electron Hamiltonian for electron $\beta$. Thus any polarising effect on the autoionising state by the nearness of the scattered electron is ignored in the model. We now introduce the Coulomb interaction $V_{\alpha \beta}=\left|\boldsymbol{r}_{\alpha}-\boldsymbol{r}_{\beta}\right|^{-1}$ by separating the total Hamiltonian as $H=H_{\alpha \beta}+V_{\alpha \beta}$. The scattering state $\left|\boldsymbol{k}_{\alpha} f^{P-}\right\rangle$ can then be expressed in terms of the asymptotic scattering state $\left|\boldsymbol{k}_{\alpha} f\right\rangle$ and powers of the interaction potential $V_{\alpha \beta}$. The first matrix element of (4.1), describing decay of the autoionising state and subsequent PCI becomes (van de Water et al 1981, equation (2.14)):

$$
\begin{aligned}
\left\langle\boldsymbol{k}_{\alpha} f^{P-}|P H Q| \boldsymbol{k}_{\beta} a^{Q-}\right\rangle & \\
\simeq & \left\langle f \mid \boldsymbol{k}_{\beta}\right\rangle\left\langle\left[\boldsymbol{k}_{\alpha}^{-}\right]\left|P H_{\beta} Q\right| a\right\rangle \\
& +\iint\left\langle\boldsymbol{k}_{\alpha} f \mid P V_{\alpha \beta} P\left[\boldsymbol{k}_{\alpha}^{\prime-}\right]\left[\boldsymbol{k}_{\beta}^{\prime-}\right]\right\rangle \frac{1}{E_{\mathrm{i}}-I P-\frac{1}{2} k_{\alpha}^{\prime 2}-\frac{1}{2} k_{\beta}^{\prime 2}+\mathrm{i} \varepsilon} \\
& \times\left\langle\left[\boldsymbol{k}_{\beta}^{\prime-}\right] \mid \boldsymbol{k}_{\beta}\right\rangle\left\langle\left[\boldsymbol{k}_{\alpha}^{\prime-}\right]\left|P H_{\beta} Q\right| a\right\rangle \mathrm{d} \boldsymbol{k}_{\alpha}^{\prime} \mathrm{d} \boldsymbol{k}_{\beta}^{\prime}
\end{aligned}
$$

where $\left|\left[\boldsymbol{k}_{\alpha}^{\prime-}\right]\left[\boldsymbol{k}_{\beta}^{\prime-}\right]\right\rangle$ and $\left|\left[\boldsymbol{k}_{\alpha}^{-}\right] f\right\rangle$ are eigenstates of $P H_{\alpha \beta} P$, and $\left|\left[\boldsymbol{k}_{\alpha}^{-}\right]\right\rangle$is a scattering state of momentum $\boldsymbol{k}_{\alpha}$ in which electron $\alpha$ only interacts with the ionic core and not with electron $\beta$. The first term on the right-hand side of (4.2) contains the amplitude for decay of the autoionising state:

$$
\left\langle\left[\boldsymbol{k}_{\alpha}^{-}\right]\left|P H_{\beta} Q\right| a\right\rangle=\left(\Gamma_{L_{\mathrm{a}}}\left(k_{\alpha}\right) / 2 k_{\alpha}\right)^{1 / 2} Y_{L_{\mathrm{a}}}^{M_{\mathrm{a}}}\left(\hat{k}_{\alpha}\right)
$$

and the overlap integral between initial and final state of the scattered electron:

$$
\left\langle f \mid \boldsymbol{k}_{\beta}\right\rangle=\int\langle f \mid \boldsymbol{r}\rangle\left\langle\boldsymbol{r} \mid \boldsymbol{k}_{\beta}\right\rangle \mathrm{d} \boldsymbol{r} .
$$

Expansion of (4.4) into partial waves results in a radial integral equivalent to the shake down model (King et al 1975). The second term in (4.2) explicitly contains the interaction $V_{\alpha \beta}$ between both electrons and includes angular momentum exchange during PCI.

The partial wave expansion given by van de Water et al (1981) of both terms in (4.2) also incorporates the excitation matrix element $\left\langle\boldsymbol{k}_{\beta} a^{Q-}|Q H P| \boldsymbol{k}_{\mathrm{i}} 0^{P+}\right\rangle$. This matrix element can be written as the product of an angle dependent part and a $T$ matrix element $T\left(k_{\beta} l_{\beta}, a L_{\mathrm{a}} \leftarrow k_{\mathrm{i}} l_{\mathrm{i}}, 0\right)$.

The present calculations were done for the case of PCI via the $\mathrm{He}^{* *}\left(2 \mathrm{p}^{2}\right)^{1} \mathrm{D}$ autoionising state and with similar approximations to those applied by van de Water et al (1981). The experimental position $E^{* *}=59.90 \mathrm{eV}$ and width $\Gamma=0.072 \mathrm{eV}$ of the $\mathrm{He}^{* *}\left(2 \mathrm{p}^{2}\right)^{1} \mathrm{D}$ autoionising state are used in the calculations. The energy variation of 
the excitation of the autoionising state, for instance due to resonances, is not taken into account. This amounts to the assumption that the excitation amplitude $T\left(k_{\beta} l_{\beta}, a L_{\mathrm{a}} \leftarrow k_{\mathrm{i}} l_{\mathrm{i}}, 0\right)$ is a step function at $E_{\mathrm{i}}=E^{* *}$ with constant absolute value and phase. Because of the considerable computing time needed, the calculations are restricted to a situation where only one partial wave contributes to the excitation of the autoionising state, i.e. immediately after the excitation of the autoionising state the scattered electron is assumed to recede with $l_{\mathrm{sc}}=0$. As a consequence in our calculations $P$ and $D$ singly excited states are only excited via angular momentum exchange during PCI. Based on the work of Lipsky and Conneely (1976) the amplitude for decay of the autoionising state, $\left(\Gamma_{L_{\mathrm{a}}}\left(k_{\alpha}\right) / 2 k_{\alpha}\right)^{1 / 2}$, is approximated by a step function at $k_{\alpha}=1$ au (see van de Water et al 1981).

Figures $10(a)$ and $(b)$ show the phase and absolute value squared, respectively, of the PCI amplitude $T_{\text {opt }}$ for excitation of $3 \mathrm{~S}, 4 \mathrm{~S}$ and $5 \mathrm{~S}$ singly excited states as a function of the residual energy of the scattered electron immediately after the excitation of the autoionising state. Thus in the absence of interference with the direct excitation of singly excited states the PCI structures would have the appearance of the curves in figure $10(b)$. As expected the PCI amplitude is largest between 0 and $1 \mathrm{eV}$ above the threshold of the autoionising state and rapidly decreases above $1 \mathrm{eV}$. The range over which the PCI phase varies increases with the principal quantum number $n$, so more complicated interference structures are to be expected in the excitation of states with increasing $n$. Clearly the apparent shift of PCI structures in excitation functions with increasing $n$ is a pure quantum mechanical effect, as there is hardly any shift in the maximum of the PCI amplitude in figure 10(b). Rather the greater and more rapid increase of the PCI phase in figure $9(a)$ gives rise to the changing PCI profiles observed in states with increasing $n$. Figure $10(\mathrm{c})$ shows the absolute value (or strength, as defined by van de Water et al 1981, equation (5.5)) of the PCI amplitude for excitation of $4 \mathrm{~S}, 4 \mathrm{P}$ and $4 \mathrm{D}$ singly excited states. Clearly the probability for angular momentum
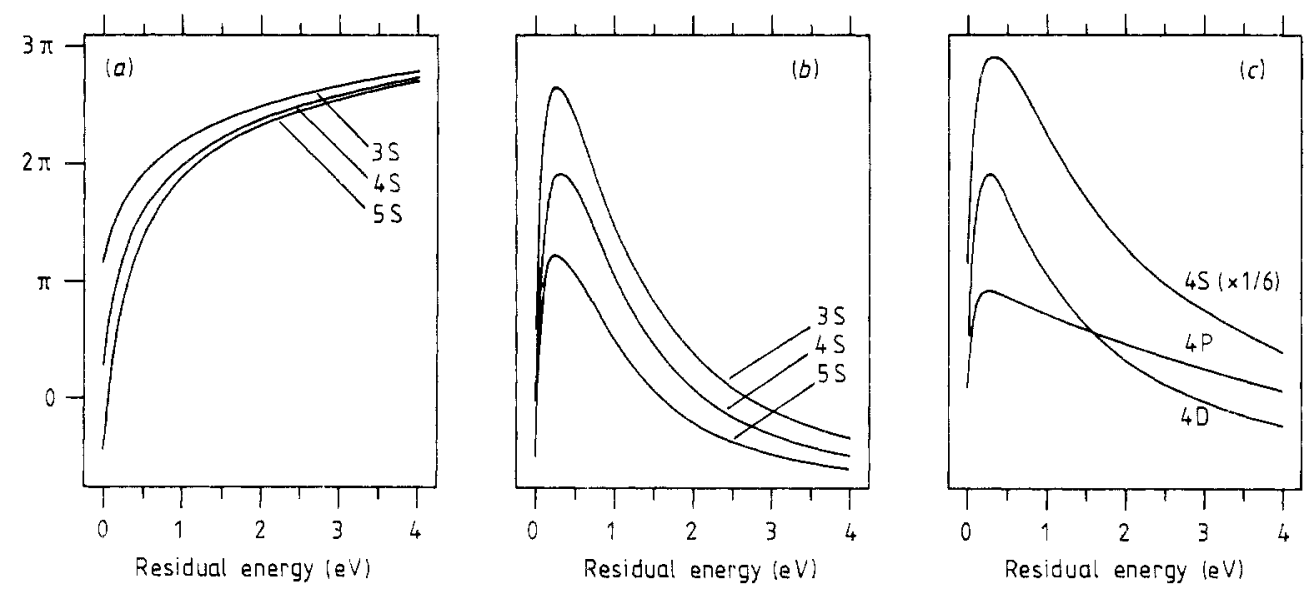

Figure 10. (a) Phase of the $\mathrm{PCl}$ amplitude related to the $\mathrm{He}^{* *}\left(2 \mathrm{p}^{2}\right)^{1} \mathrm{D}$ state for excitation of $n=3,4,5$ and $L=0$ singly excited states. (b) Absolute value squared of the PCI amplitude for excitation of $n=3,4,5$ and $L=0$ singly excited states. For all curves the same vertical scale is used. (c) Absolute value of the PCI amplitude for $n=4$ and $L=0$, 1,2 singly excited states. Vertical scales are the same, taking into account that the $L=0$ curve is reduced by a factor of 6 . Clearly the probability for angular momentum exchange during $\mathrm{PCI}$ is small in this case. 
exchange during PCI is rather small, but increases somewhat at higher residual energies. Van de Water et al (1981) have calculated probabilities for angular momentum exchange in other cases and conclude that angular momentum exchange may be more important in situations where the scattered electrons recede anisotropically, with $l_{\text {sc }} \neq 0$, after having excited the autoionising state. Experimental evidence for angular momentum exchange during PCI was given by van der Burgt et al (1985a) for a situation where the scattered electron initially recedes with $l_{\mathrm{sc}}=2$.

We have fitted the calculated PCI amplitudes to the measurements by using only one adjustable parameter (see also Morgenstern et al 1977). It has been argued by Read and Comer (1980) that the successful fitting of PCI profiles might partly be due to the large number of variable parameters in the fitting formula. Moreover in our calculations, and also in the previous models for $\mathrm{PCI}$, the excitation of the autoionising state is not taken into account. In view of these considerations we have only used the phase of the direct excitation background as a variable parameter in the fits. The interfering part of the direct excitation background was arbitrarily assumed to be much greater than the PCI amplitude. Figures 11(a), $(b)$ and $(c)$ show the results of the one-parameter fits. The values $0.8 \pi, 0.3 \pi$ and $1.6 \pi$ of the background phase were chosen such that a close resemblance between the $5 \mathrm{~S}, 5 \mathrm{P}$ and $5 \mathrm{D}$ calculated spectra and the $5^{1} \mathrm{~S}, 5^{3} \mathrm{P}$ and $5^{1} \mathrm{D}$ measured spectra was achieved. Thereupon the $n=3$ and $n=4$ spectra were calculated, using the same values for the background phase. Thus we assume the background phase to be approximately independent of the principal quantum number $n$ for each series of spectra. This seems a legal approximation as the energy of the inelastically scattered electrons is much higher than the energy difference between the singly excited states of different principal quantum number. A close resemblance with the measurements is observed except for the $3^{3} \mathrm{P}$ spectrum where cascading from higher ${ }^{3} \mathrm{~S}$ levels is significant $(\$ 3.2)$. Comparison with the ${ }^{1} \mathrm{D}$ series is somewhat hampered by the curved background present in the measured spectra. The narrow peak in the calculations just above the threshold of the autoionising state is less clear in the measurements, maybe due to insufficient resolution of the incident electron beam and to the fact that we have approximated the excitation of the autoionising state by a step function.

We have not been able to find an acceptable fit of the PCI structure at $59.90 \mathrm{eV}$ in the $4^{3} \mathrm{~S}$ excitation function. A value of $0.9 \pi$ of the background phase results in a good fit of the higher part of the PCI structure, above $60.5 \mathrm{eV}$, but the lower part of the fitted profile then shows a peak shifted over $0.2 \mathrm{eV}$ and reduced to half the height as compared with the peak observed in the measurement. This supports the suggestion of Heideman (1980) that the threshold cross section for excitation of the $\mathrm{He}^{* *}\left(2 \mathrm{p}^{2}\right)^{1} \mathrm{D}$ autoionising state is enhanced by a resonance.

Despite the qualitative nature of the calculations some conclusions can be drawn. The structures observed above $59.90 \mathrm{eV}$ in the $3^{1} \mathrm{~S}$ and $3^{1} \mathrm{D}$ excitation functions may at first sight be considered to be caused by the presence of broad negative ion resonances, which decay directly to the singly excited states. The close resemblance with the calculated PCI profiles for $3 \mathrm{~S}$ and $3 \mathrm{D}$ suggests that these structures are rather caused by $\mathrm{PCI}$ via the $\mathrm{He}^{* *}\left(2 \mathrm{p}^{2}\right)^{1} \mathrm{D}$ autoionising state. In the other $n=3$ excitation functions broad structures are also observed, most notably in the $3^{3} \mathrm{P}$ excitation function above $57.82 \mathrm{eV}$. Here two narrow Beutler-Fano resonance profiles are observed superimposed on a broad dip, the latter likely being caused by PCI via the $\mathrm{He}^{* *}\left(2 \mathrm{~s}^{2}\right)^{1} \mathrm{~S}$ and $\mathrm{He}^{* *}(2 \mathrm{~s} 2 \mathrm{p})^{3} \mathrm{P}$ autoionising states. Superimposed PCI profiles due to these states occur clearly in the $4^{3} \mathrm{P}$ and $5^{3} \mathrm{P}$ excitation functions. An important aspect of the post-collision 

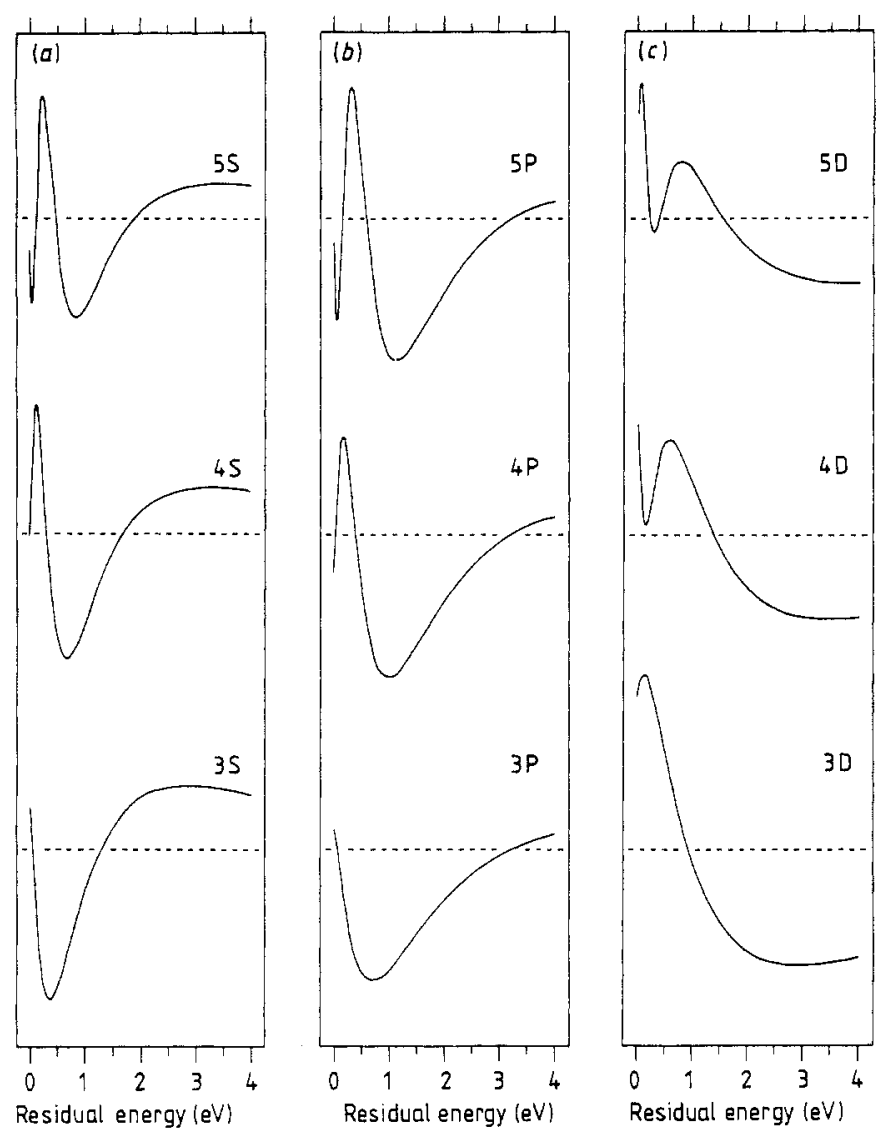

Figure 11. (a) Calculated profiles of PCI structures related to the $\mathrm{He}^{* *}\left(2 \mathrm{p}^{2}\right)^{1} \mathrm{D}$ autoionising state in the $n=3,4,5$ and $L=0$ excitation functions. The interfering part of the background-indicated by a broken line-was arbitrarily chosen much greater than the PCI amplitude. The phase of the background was chosen such that a close resemblance with the $n^{1} \mathrm{~S}$ excitation functions was obtained. (b) Calculated PCI profiles in the $n=3,4,5$ and $L=1$ excitation functions. Compare with the $n^{3} \mathrm{P}$ excitation functions in figure 6 . (c) Calculated PCI profiles in the $n=3,4,5$ and $L=2$ excitation functions. Compare with the $n^{1} \mathrm{D}$ excitation functions in figure 7.

interaction is the large energy range over which PCI excitation of singly excited states is possible. The calculations show still significant $\mathrm{PCI}$ excitation up to $4 \mathrm{eV}$ above the threshold of the $\mathrm{He}^{* *}\left(2 \mathrm{p}^{2}\right)^{1} \mathrm{D}$ autoionising state. This is also observed in the measurements. See for instance the ${ }^{1} \mathrm{~S}$ spectra: both the measurements and the calculations show a broad maximum in the PCI profile at $62 \mathrm{eV}$. Also the PCI profiles in the ${ }^{3} \mathrm{D}$ excitation functions exhibit a maximum near $62 \mathrm{eV}$. Another important aspect is the interference of the PCI excitation with the direct excitation background. The PCI structures above $59.90 \mathrm{eV}$ in the ${ }^{1} \mathrm{~S}$ excitation functions look very different from the structures in the ${ }^{1} \mathrm{D}$ excitation functions. However, an overall change of the background phase of $\pi$, approximately, would result in ${ }^{1} \mathrm{D}$ structures similar to the ${ }^{1} \mathrm{~S}$ structures. In comparing PCI structures in different excitation functions, one is tempted to look 
at the positions of peaks and dips in the profiles. Clearly interference means that great care needs to be exercised in such a comparison. It is of interest to consider the validity of the shake down model by comparing the first term of $T_{\text {opt }}$, which is equivalent to the shake down model, with the second, higher order term. In the present calculations both terms contribute to the excitation of $\mathrm{S}$ singly excited states, while $\mathrm{P}$ and $\mathrm{D}$ excitation is completely contained in the higher order term, so we can only compare both models with respect to $S$ excitation. We have found that not the shapes but only the sizes of the curves in figure $10(a)$ and $(b)$ change significantly, when the higher order term is omitted in the calculations. Removing the higher order term results in a reduction of the PCI amplitude of about $50 \%$ and a small overall decrease of the PCI phase of about $0.1 \pi$. We conclude that the shake down model gives a good qualitative description of the PCI for the case of $\mathrm{S}$ excitation.

\section{Interpretation of the results}

Prior to a systematic discussion of the excitation functions it is important to consider the question whether two overlapping PCI profiles can interfere. This is a relevant question in view of the large widths of the PCI profiles. When measuring optical excitation functions we detect the emitted photons after capture of the scattered electron into a singly excited state, but we do not obtain any information on the direction of the ejected electrons. This means that in our experiment we integrate over the angle coordinates $\theta$ and $\varphi$ of the ejected electrons. The total excitation cross section may then be written as

$$
\sigma=\iint\left(|B|^{2}+\left|A+a \mathrm{e}^{\mathrm{i} \alpha}+b \mathrm{e}^{\mathrm{i} \beta}\right|^{2}\right) \sin \theta \mathrm{d} \theta \mathrm{d} \varphi
$$

where $a \mathrm{e}^{\mathrm{i} \alpha}$ and $b \mathrm{e}^{\mathrm{i} \beta}$ refer to the contributions from the two autoionising states, respectively, and $A$ and $B$ to the interfering and non-interfering parts, respectively, of the direct excitation. Expanding the dependences of $A, a \mathrm{e}^{\mathrm{i} \alpha}$ and $b \mathrm{e}^{\mathrm{i} \beta}$ on $\theta$ and $\varphi$ in spherical harmonics $Y_{l}^{m}$ immediately shows that all interference terms except those with the same $l$ and $m$ for $A, a \mathrm{e}^{\mathrm{i} \alpha}$ and $\mathrm{e}^{\mathrm{i} \beta}$ vanish due to orthogonality of the spherical harmonics.

In the case of helium the angular momentum $l$ of the ejected electron is equal to the orbital angular momentum of the autoionising state, as the ground state of the $\mathrm{He}^{+}$ion has zero orbital angular momentum. It follows that in the absence of angular momentum exchange during PCI autoionising states with different angular momenta are not expected to interfere in the optical excitation functions.

\subsection{A selection rule for threshold excitation of autoionising states}

In $\S 4$ it was tacitly assumed that above $59.90 \mathrm{eV}$ only the $\mathrm{He}^{* *}\left(2 \mathrm{p}^{2}\right)^{1} \mathrm{D}$ state contributes significantly to the PCI profiles in the excitation functions and that the $\mathrm{He}^{* *}(2 \mathrm{~s} 2 \mathrm{p})^{1} \mathrm{P}$ state is not or only very weakly excited. As the oscillations of an arbitrary PCr profile are sharp near threshold and become broader with increasing incident energy, the presence of the $\mathrm{He}^{* *}(2 \mathrm{~s} 2 \mathrm{p})^{1} \mathrm{P}$ state would be recognised easily by a sharp structure at $60.13 \mathrm{eV}$, superimposed on the PCI profile of the $\mathrm{He}^{* *}\left(2 \mathrm{p}^{2}\right)^{1} \mathrm{D}$ state. Weak indications for superimposed $\mathrm{PCI}$ profiles are seen in the $5^{1} \mathrm{~S}, 6^{1} \mathrm{~S}$ and $4^{1} \mathrm{P}$ excitation functions only. The $\mathrm{He}^{* *}(2 \mathrm{~s} 2 \mathrm{p})^{1} \mathrm{P}$ state is clearly absent at $60.13 \mathrm{eV}$ in the ${ }^{3} \mathrm{~S}$ excitation functions. 
The structures around $60 \mathrm{eV}$ in the ${ }^{3} \mathrm{P},{ }^{1} \mathrm{D}$ and ${ }^{3} \mathrm{D}$ excitation functions clearly have their onset at $59.90 \mathrm{eV}$ and there is no indication for a superposition of structures. We conclude that the $\mathrm{He}^{* *}(2 \mathrm{~s} 2 \mathrm{p})^{1} \mathrm{P}$ autoionising state is not or only very weakly excited near its threshold.

Also the $\mathrm{He}^{* *}\left(2 \mathrm{p}^{2}\right)^{1} \mathrm{~S}$ and $\mathrm{He}^{* *}(2 \mathrm{~s} 3 \mathrm{~s})^{3} \mathrm{~S}$ autoionising states at 62.06 and $62.62 \mathrm{eV}$, respectively, are very weakly or not at all observed in our measurements. Above $62.7 \mathrm{eV}$ the rapidly increasing number of autoionising states cannot be resolved in our experiment. Moreover the incident energy scale, which was calibrated on the $\mathrm{He}^{-}\left(2 s^{2} 2 p\right)^{2} \mathrm{P}$ resonance at $57.22 \mathrm{eV}$, may have shifted by some tens of meV near $63 \mathrm{eV}$. We note however, that the $\mathrm{He}^{* *}(23 \mathrm{sp}-)^{1} \mathrm{P}$ autoionising state is not observed in the ejected electron spectra of Hicks and Comer (1975) which were measured with a resolution of about $40 \mathrm{meV}$. We therefore attribute the structures observed around $63 \mathrm{eV}$ in the $4^{3} \mathrm{P}$ and $5^{3} \mathrm{P}$ excitation functions to the $\mathrm{He}^{* *}(2 \mathrm{~s} 3 \mathrm{~s})^{1} \mathrm{~S}$ and $\mathrm{He}^{* *}(23 \mathrm{sp}+)^{3} \mathrm{P}$ states at 62.94 and $63.07 \mathrm{eV}$. The structure having its onset near $63.50 \mathrm{eV}$ clearly is to be ascribed to the $\mathrm{He}^{* *}(2 \mathrm{p} 3 \mathrm{p})^{1} \mathrm{D}$ state.

Comparing the autoionising states observed in our excitation functions with the supermultiplet classification scheme of Lin (1984) in table 1 , it is easily seen that only states with quantum numbers $(K, T)^{A}=(1,0)^{+}$are significantly present in the excitation functions. As the ground state of helium is classified as ${ }_{1}(0,0){ }_{1}^{+1} S^{e}$ we find a selection rule

$$
\Delta K=1 \quad \Delta T=0 \quad \text { and } \quad \Delta A=0
$$

for the near threshold excitation of autoionising states in e-He scattering. This selection rule is related to near threshold electron impact excitation of autoionising states. At higher incident electron energies a much weaker selection rule $\Delta A=0$ holds. This can be verified by looking at the autoionising states observed in the ejected electron spectra of Hicks and Comer (1975).

Table 1 shows that the states with $(K, T)^{A}=(1,0)^{+}$occur in distinct groups containing ${ }^{1} \mathrm{~S}^{\mathrm{e}},{ }^{3} \mathrm{P}^{\circ}$ and ${ }^{1} \mathrm{D}^{\mathrm{e}}$ states. The states of each group form a rotor series (Lin 1984), and may be interpreted, in terms of the collective rovibrational model of Kellman and Herrick (1980), as different rotational modes of a particular vibrational mode with the correlation quantum numbers $(1,0)^{+}$. The group between 57.82 and $62 \mathrm{eV}$ is the lowest vibrational member of the $(1,0)^{+}$mode. Apparently the other vibrational modes, $(-1,0)^{+},(0,1)^{+}$and $(1,0)^{-}$which lie in the energy range covered, are not, or very weakly, excited in our experiment. Based on the selection rule we conclude that the autoionising states observed in the excitation functions occur in distinct groups, each group being labelled by the quantum numbers ${ }_{n}(1,0)_{2}^{+}$. The appearance of the $n=2$, 3 and 4 groups can be seen most clearly in the $5^{1} \mathrm{~S}, 6^{1} \mathrm{~S}, 4^{3} \mathrm{P}$ and $5^{3} \mathrm{P}$ excitation functions. This is in complete analogy with the measurements of Buckman et al (1983), where distinct groups of resonances appear in the cross section for electron impact excitation of metastable helium atoms.

\subsection{Shape resonances close to the thresholds of the autoionising states}

By measuring the strength of the $59.90 \mathrm{eV}$ structure in the ${ }^{3} \mathrm{~S}$ excitation functions Heideman (1980) suggested a resonance like behaviour of the threshold excitation cross section of the $\mathrm{He}^{* *}\left(2 \mathrm{p}^{2}\right)^{\mathrm{l}} \mathrm{D}$ autoionising state. Recently van der Burgt et al (1985b) have found experimental evidence for the presence of a negative ion resonance at $59.90 \mathrm{eV}$ in ejected electron spectra. The effect of this resonance can be 
seen by comparing the $59.90 \mathrm{eV}$ structures in the ${ }^{3} \mathrm{~S}$ excitation functions in figure 4 with the structures in the ${ }^{3} \mathrm{P}$ excitation functions in figure 6 . In the ${ }^{3} \mathrm{P}$ excitation functions interference structures are observed which have their onset (not their peaks) at $59.90 \mathrm{eV}$ (note the small dip at $59.90 \mathrm{eV}$ in the $5^{3} \mathrm{P}$ excitation function), and which show a small shift to higher energies with the increase of the principal quantum number. These structures could well be fitted with our PCI model, see $\S 4$.

The structure observed above $59.90 \mathrm{eV}$ in the $4^{3} \mathrm{~S}$ and $5^{3} \mathrm{~S}$ excitation functions is exceptional, not only because of the height of the peak but even more because of the position of the peak precisely at the threshold of the $\mathrm{He}^{* *}\left(2 \mathrm{p}^{2}\right)^{1} \mathrm{D}$ autoionising state. This structure is not due to direct decay of a resonance to the ${ }^{3} \mathrm{~S}$ singly excited states as only a very weak structure at the same energy is observed in the $3^{3} \mathrm{~S}$ excitation function. Post-collision interaction is involved, as the profile extends to $62 \mathrm{eV}$ and exhibits a small shift to higher energies with the increase of the principal quantum number, but we could not find an acceptable fit to the PCI model of $\S 4$. Therefore we attribute the structure in the ${ }^{3} \mathrm{~S}$ excitation functions to a $\mathrm{He}^{-}$shape resonance which strongly enhances the excitation cross section of the $\mathrm{He}^{* *}\left(2 \mathrm{p}^{2}\right)^{1} \mathrm{D}$ autoionising state in a narrow range just above its threshold. Based on the estimated width of the resonance (van der Burgt et al 1985b) this range is of the order of $500 \mathrm{meV}$.

As the resonance is observed only in the ${ }^{3} \mathrm{~S}$ ( and maybe also ${ }^{1} \mathrm{~S}$ ) excitation functions it occurs apparently only in the $l_{\mathrm{sc}}=0$ partial wave of the scattered electron, assuming that no angular momentum exchange occurs during PCI. Therefore the resonance is probably a ${ }^{2} \mathrm{D}$ state and can not be one of the higher lying $\mathrm{He}^{-}\left(2 \mathrm{~s} 2 \mathrm{p}^{2}\right)^{2} \mathrm{~S}$ or $\mathrm{He}^{-}\left(2 \mathrm{p}^{3}\right)^{2} \mathrm{P}$ intrashell resonances. We assume that this resonance is of the intershell type discussed in $\S 2.3$. Using the notation of Nesbet (1978) the (approximate) configuration of the resonance would then be $\mathrm{He}^{-}\left(2 \mathrm{p}^{2} \overline{\mathrm{s}}\right)^{2} \mathrm{D}$, i.e. an s electron weakly bound in the polarisation potential of the $\mathrm{He}^{* *}\left(2 \mathrm{p}^{2}\right)^{1} \mathrm{D}$ autoionising state.

The experiments show that the ${ }^{1} \mathrm{~S}^{\mathrm{e}}$ and ${ }^{3} \mathrm{P}^{\mathrm{o}}$ members of the ${ }_{2}(1,0)_{2}^{+}$rotor series are also excited strongly near their thresholds by shape resonances. The $4^{1} \mathrm{D}$ excitation function exhibits a structure at $58.30 \mathrm{eV}$ of similar appearance to the $59.90 \mathrm{eV}$ structure in the $4^{3} \mathrm{~S}$ excitation function. Also in the $3^{1} \mathrm{P}$ excitation function such a structure occurs at $58.30 \mathrm{eV}$. These structures are clearly related to excitation and decay of the $\mathrm{He}^{* *}(2 \mathrm{~s} 2 \mathrm{p})^{3} \mathrm{P}$ autoionising state followed by the post-collision interaction, as the structures extend over $2 \mathrm{eV}$ (at least) and shift to higher energies in the $5^{1} \mathrm{D}$ and $4^{1} \mathrm{P}$ excitation functions, respectively. The narrow peak seen at the threshold of the $\mathrm{He}^{* *}(2 \mathrm{~s} 2 \mathrm{p})^{3} \mathrm{P}$ state indicates a high threshold excitation cross section which we also attribute to the presence of a shape resonance. The sharp rise in the excitation cross section of the $\mathrm{He}^{* *}(2 \mathrm{~s} 2 \mathrm{p})^{3} \mathrm{P}$ autoionising state was already reported by Spence (1975) and also Roy et al (1978b) found some evidence for the presence of a resonance in the $\mathrm{He}^{* *}(2 \mathrm{~s} 2 \mathrm{p})^{3} \mathrm{P}$ excitation cross section. The question arises of whether the shape resonance at the $\mathrm{He}^{* *}(2 \mathrm{~s} 2 \mathrm{p})^{3} \mathrm{P}$ threshold is the $\mathrm{He}^{-}\left(2 \mathrm{~s} 2 \mathrm{p}^{2}\right)^{2} \mathrm{D}$ intrashell resonance, or both an intrashell and an intershell resonance occur near to $58.30 \mathrm{eV}$. A more detailed discussion on this matter will be given in $\S 6$.

The strong peaks observed near $57.8 \mathrm{eV}$ in the $4^{3} \mathrm{P}$ and $5^{3} \mathrm{P}$ excitation functions show that also the $\mathrm{He}^{* *}\left(2 \mathrm{~s}^{2}\right)^{1} \mathrm{~S}$ autoionising state is excited strongly at its threshold, probably due to an intershell resonance of the configuration $\mathrm{He}^{-}\left(2 \mathrm{~s}^{2} \overline{\mathrm{p}}\right)^{2} \mathrm{P}$. It may be argued that such a configuration with one electron outside a closed-shell core can not be stable. However, such a reasoning relies on the independent-electron model for the classification of atomic states. When electron-electron correlations are accounted for, two ${ }^{1} \mathrm{~S}$ states are found with both electrons in the $n=2$ shell, one with a large 
probability to find the two electrons at opposite sides and equal distances from the nucleus and another where the two electrons occupy a much larger domain of configuration space (see figure 6 of Lin 1982). The former is expected to have a fairly large polarisability, so that a potential well may be formed deep enough to bind a third electron.

Roy et al (1978a) studied the PCI excitation by observing the inelastically scattered electrons. In the $n=4$ differential excitation curves they observed structures of a similar appearance to our structures described above. The presence of intershell resonances in the lowest rotor series raises the question whether also intershell resonances exist near the thresholds of autoionising states in the higher rotor series. As autoionising states within the same $(1,0)_{2}^{+2 S+1} L^{\pi}$ channel are characterised by similar correlated motion of the two electrons, we expect that intershell resonances related to higher parent states within the same channel have the same configuration and therefore appear in the same excitation function. It has already been noted (at the end of $\S 5.1$ ) that autoionising states in higher $(n \geqslant 3)$ rotor series of the ${ }_{n}(1,0)_{2}{ }^{2 S+1} L^{\pi}$ channel are also strongly excited near their thresholds, indicating the presence of resonances near their thresholds. Clear evidence for this is seen in the $4^{3} \mathrm{P}$ excitation function. At $62.94 \mathrm{eV}$ a narrow peak is observed at the threshold of the $\mathrm{He}^{* *}(2 \mathrm{~s} 3 \mathrm{~s})^{1} \mathrm{~S}$ autoionising state probably due to a resonance of the configuration $\mathrm{He}^{-}(2 \mathrm{~s} 3 \mathrm{~s} \overline{\mathrm{p}})^{2} \mathrm{P}$. Baxter et al $(1979 \mathrm{~b})$ also report the presence of a resonance at this energy. Other examples are less clear due to insufficient resolution of the incident electron beam. In the $4^{1} \mathrm{D}$ excitation function a weak asymmetric structure is present at $63 \mathrm{eV}$ (the peak at the ${ }^{3} \mathrm{P}^{\circ}$ threshold is barely resolved) similar to the asymmetric structure above the ${ }^{3} \mathrm{P}^{\circ}$ threshold at $58.30 \mathrm{eV}$. In the $5^{3} \mathrm{~S}$ excitation function a narrow peak is observed just above the ${ }^{1} \mathrm{D}^{\mathrm{e}}$ threshold at $63.50 \mathrm{eV}$, similar to the peak just above the ${ }^{1} \mathrm{D}^{\mathrm{e}}$ threshold at $59.90 \mathrm{eV}$. Finally we note that in the $2^{1} \mathrm{~S}$ and $2^{3} \mathrm{~S}$ excitation curves only the $\mathrm{He}^{-}\left(2 \mathrm{~s}^{2} 2 \mathrm{p}\right)^{2} \mathrm{P}$ and $\mathrm{He}^{-}\left(2 \mathrm{~s} 2 \mathrm{p}^{2}\right)^{2} \mathrm{D}$ intrashell resonances are prominently present. In the $2^{3} \mathrm{~S}$ curve a small structure is seen that could possibly be due to PCI via the $\mathrm{He}^{* *}\left(2 \mathrm{p}^{2}\right)^{1} \mathrm{D}$ state. This is in accordance with our expectation expressed at the end of $\S 2.3$.

\subsection{The polarisability of doubly excited states}

We have pointed already to a relationship between the polarisability of a doubly excited state and the presence of an intershell resonance close to its threshold. To see which states have a large polarisability and which states have not, it is helpful to look at the surface density plots of Lin (1982). The polarisation of a doubly excited state may be visualised by a decrease of amplitude around the Wannier point $\alpha=$ $\tan ^{-1}\left(r_{2} / r_{1}\right)=\pi / 4$ and $\theta_{12}=\cos ^{-1}\left(\hat{r}_{1} \cdot \hat{r}_{2}\right)=\pi$ and an increase of amplitude towards smaller $\theta_{12}$. Therefore we expect that those doubly excited states which have a small amplitude outside the Wannier region have the highest polarisability.

We consider the various channels to which the autoionising states in table 1 belong. In figure 6 of Lin (1982) plots of the surface charge distribution for the $\mu=2 A$ and $\mu=2 B$ channels of $\mathrm{H}^{-}\left({ }^{1} \mathrm{~S}^{\mathrm{e}}\right)$ are shown. The surface charge distributions for $\mathrm{He}\left({ }^{1} \mathrm{~S}^{e}\right)$ have a similar appearance. The $\mu=2 A$ and $\mu=2 B$ channels correspond to the $\mu=(1,0)_{2}^{+1} S^{e}$ and $\mu=(-1,0)_{2}^{+1} S^{e}$ channels of Lin (1984), respectively. Doubly excited states that have identical $(K, T)^{A}$ quantum numbers are isomorphic (Lin 1984), so the $(1,0)_{2}^{+1} \mathrm{~S}^{\mathrm{e}},(1,0)_{2}^{+3} \mathrm{P}^{\circ}$ and $(1,0)_{2}^{+1} \mathrm{D}^{\mathrm{e}}$ channels have similar correlation patterns. A surface charge distribution plot of the $(0,1)_{2}^{+1} \mathrm{P}^{\circ}$ channel of helium is seen in figure 6 of Le Dourneuf and Watanabe (1984). 
It is seen that the $(1,0)_{2}^{+}$surface charge distribution has a large amplitude in and a very small one outside the Wannier region and thus meets the above posed requirements for a high polarisability; indeed these states occur very prominently in our measured excitation functions. On the other hand the $(-1,0)_{2}^{+}$and $(0,1)_{2}^{+}$surface charge distributions are more spread out over the whole $\left(\alpha, \theta_{12}\right)$ plane and doubly excited states with $(-1,0)_{2}^{+}$and $(0,1)_{2}^{+}$quantum numbers therefore are only weakly polarisable. Accordingly these states do not support intershell resonances near their threshold and are not, or weakly, observed in the excitation functions.

This also explains why the selection rule $\Delta K=1, \Delta T=0$, mentioned earlier, applies only close to the thresholds of the autoionising states. The additional part of the selection rule: $\Delta A=0$ holds more generally for electron impact excitation of doubly excited states. In a classical picture $A=+1$ means that both electrons orbit in opposite senses around the nucleus (Lin 1984). To excite $A=-1$ states from the $\mathrm{He}_{1}(0,0)_{1}^{+1} \mathrm{~S}^{\mathrm{e}}$ ground state one of the electrons has to change its sense of rotation. This is much harder than exciting an $A=+1$ state in which both electrons keep their original opposite sense of rotation (Lin 1984).

The above explanation of the selection rule and the occurrence of resonances in terms of surface charge distributions is of course of a qualitative nature. We hope that more theoretical work will be done in the near future along the lines of three-electron correlations (see Clark and Green 1980, Watanabe et al 1982).

\section{Discussion}

In the previous sections we have made a distinction between two classes of resonances in the autoionisation region, mainly based on their different decay modes. Resonances of the intrashell type-like the $\mathrm{He}^{-}\left(2 \mathrm{~s}^{2} 2 \mathrm{p}\right)^{2} \mathrm{P}$ resonance at $57.22 \mathrm{eV}$-were found to decay directly to the singly excited states, and to occur most prominently in the cross sections of the lower $n$ singly excited states (in particular $n=2$ ). Resonances of the intershell type-like the shape resonances at the 57.82 and $59.90 \mathrm{eV}$ autoionisation thresholds-were found to enhance the threshold excitation cross sections of their parent autoionising states strongly. These resonances are observed in the cross sections of the larger $n$ singly excited states via the post-collision interaction. The approximate configurations of these intershell resonances could be found because they appear only prominently in excitation functions of singly excited states of a particular orbital angular momentum.

This empirical distinction between two classes of resonances is also seen in the polarisation measurements of Defrance (1980). It is seen in his measurements that only the intrashell resonances give rise to clear structures in the polarisation of helium lines in the autoionisation region. As the intershell resonances decay via PCI mainly to singly excited states of a particular angular momentum, these resonances will probably not be observed in a summation of cross sections of singly excited states of various angular momenta, as in the trapped electron experiments of Spence (1980). Analysis of structures at $58.30 \mathrm{eV}$ in the excitation functions is very complicated. In the first place structures at $58.30 \mathrm{eV}$ may arise through a superposition of the PCI profiles of the $\mathrm{He}^{* *}\left(2 \mathrm{~s}^{2}\right)^{1} \mathrm{~S}$ and $\mathrm{He}^{* *}(2 \mathrm{~s} 2 \mathrm{p})^{3} \mathrm{P}$ autoionising states. Secondly structures around $58.30 \mathrm{eV}$ may be affected by resonances via various mechanisms. (i) The presence of a shape resonance is indicated by structures in both the $4^{1} \mathrm{D}$ and $3{ }^{1} \mathrm{P}$ excitation functions, similar to the $59.90 \mathrm{eV}$ structure in the $4^{3} \mathrm{~S}$ excitation function. 
(ii) The $\mathrm{He}^{-}\left(2 \mathrm{~s} 2 \mathrm{p}^{2}\right)^{2} \mathrm{D}$ intrashell resonance gives rise to a Beutler-Fano resonance profile at $58.30 \mathrm{eV}$ in the excitation of the $n=2$ states (figure 9). (iii) The excitation cross section of the $\mathrm{He}^{* *}\left(2 \mathrm{~s}^{2}\right)^{1} \mathrm{~S}$ autoionising state is enhanced by a negative ion resonance (presumably the $\mathrm{He}^{-}\left(2 \mathrm{~s} 2 \mathrm{p}^{2}\right)^{2} \mathrm{D}$ intrashell resonance) at $0.48 \mathrm{eV}$ above its threshold. Via $\mathrm{PCI}$ this may give rise to additional structure at $58.30 \mathrm{eV}$.

Based on these points one gets the impression that both an intrashell and an intershell resonance occur close to $58.30 \mathrm{eV}$. This impression is supported by a comparison of the ${ }^{1} \mathrm{D}$ and ${ }^{3} \mathrm{D}$ excitation functions. The $58.30 \mathrm{eV}$ structure in the $4^{1} \mathrm{D}$ excitation function is clearly part of a PCI profile which extends to $60 \mathrm{eV}$ and which exhibits a shift towards higher energy in the $5^{1} \mathrm{D}$ excitation function. We think that the double peak in the $5^{1} \mathrm{D}$ excitation function is just a PCI effect, because the PCI phase (as a function of incident energy) features a greater increase for PCI excitation of singly excited states of higher $n$ (see figure $10(a)$ ). The narrow peak at the $\mathrm{He}^{* *}(2 \mathrm{~s} 2 \mathrm{p})^{3} \mathrm{P}$ threshold shows a high threshold excitation which we attribute to the presence of an intershell resonance. The $58.30 \mathrm{eV}$ structures in the ${ }^{3} \mathrm{D}$ excitation functions have a very different appearance. PCI profiles extending to $60 \mathrm{eV}$ are present, but are much smaller than the prominent peaks at $58.30 \mathrm{eV}$. As the $\mathrm{He}^{-}\left(2 \mathrm{~s}^{2} 2 \mathrm{p}\right)^{2} \mathrm{P}$ resonance also occurs strongly in the ${ }^{3} \mathrm{D}$ excitation functions, we think that the $58.30 \mathrm{eV}$ peaks in the ${ }^{3} \mathrm{D}$ excitation functions are for the larger part due to direct decay of an intrashell resonance.

Theoretical work is not conclusive on this matter. Several theoretical calculations support the existence of a ${ }^{2} \mathrm{D}$ Feshbach resonance just below the $\mathrm{He}^{* *}(2 \mathrm{~s} 2 \mathrm{p})^{3} \mathrm{P}$ threshold. However, according to Chung (1980) a resonance can not be formed, because the $(2 s 2 p)^{3} \mathrm{P}$ core appeared to be repulsive to a third $\mathrm{p}$ electron when a Feshbach resonance was searched for variationally.

A model for post-collision interaction in which resonances play a dominant role was put forward by Nesbet (1976). He suggested that the electron impact excitation of autoionising states of helium mainly takes place via negative ion resonances. Assuming angular momentum exchange during PCI to be negligible, this model leads to $L$-selective excitation of singly excited states via mechanism (iii). Nesbet (1976) only considered the four intrashell resonances discussed by Fano and Cooper (1965) and calculated the positions of the upper two $\mathrm{He}^{-}\left(2 \mathrm{~s} 2 \mathrm{p}^{2}\right)^{2} \mathrm{~S}$ and $\mathrm{He}^{-}\left(2 \mathrm{p}^{3}\right)^{2} \mathrm{P}$ resonances to be at 59.5 and $60.6 \mathrm{eV}$. The widths were estimated to be 0.4 and $1.0 \mathrm{eV}$. We do not find evidence for resonances at these positions in the excitation functions.

\section{Conclusion}

We have studied excitation of singly excited helium states by detection of the yield of emitted photons as a function of the incident electron energy in the 56 to $66 \mathrm{eV}$ region. A wealth of hitherto unknown structures is observed caused by resonances and by PCI via autoionising states.

It is found that only autoionising states, belonging to a class of states with a large probability to find both electrons in the Wannier region of configuration space, are strongly excited. As a consequence the excitation functions exhibit a Rydberg series of structures formed by the PCI profiles of the subsequent groups of autoionising states.

Some of the PCI profiles show prominent peaks at the thresholds of the autoionising states belonging to this class. These structures are explained by the presence of shape resonances which strongly enhance the excitation cross sections of the autoionising states in a narrow range just above their thresholds. As the shape resonances mainly 
decay to their parent autoionising states they are not observed in the cross sections of the $n=2$ singly excited states, which are not affected by the PCI mechanism.

We have sought for an explanation of these features by pointing out that the above mentioned class of autoionising states is expected to have a high polarisability. As a result these states may be able to support a shape resonance (or virtual state) formed by an electron very weakly bound in the polarisation potential of the doubly excited core. In accordance with the measurements such shape resonances are expected to occur only very close to the thresholds of the parent autoionising states.

An optical potential model for post-collision interaction is used to calculate some of the PCI profiles. Although in this model the influence of the scattered electron on the autoionising state is ignored, the model is capable of reproducing the shapes of the PCI profiles fairly well. We find that the PCI profiles extend to about $4 \mathrm{eV}$ above the thresholds of the autoionising states, and that the PCI energy shift is a pure interference effect, caused by the change with energy of the phase of the PCI matrix element with the principal quantum number of the PCI excited singly excited state.

\section{Acknowledgments}

We gratefully acknowledge discussions with Dr C D Lin and Dr R K Nesbet and correspondence with Dr S Watanabe. We are indebted to $Y M$ Kampmeier for her assistance with the measurements. This work was performed as a part of the research programme of the 'Stichting voor Fundamenteel Onderzoek der Materie' (FOM) with financial support from the "Nederlandse Organisatie voor Zuiver Wetenschappelijk Onderzoek' (ZWO).

\section{References}

Andrick D 1979 J. Phys. B: At. Mol. Phys, 12 L175-6

Baxter J A, Comer J, Hicks P J and McConkey J W 1979a J. Phys. B: At. Mol. Phys. 12 2031-41 - 1979b J. Phys. B: At. Mol. Phys. 12 L355-8

Buckman S J, Hammond P, Read F H and King G C 1983 J. Phys. B: At. Mol. Phys. $164039-47$

van der Burgt P J M, van Eck J and Heideman H G M 1985a J. Phys. B: At. Mol. Phys. 18 999-1009

- 1985b J. Phys. B: At. Mol. Phys. 18 L171-4

van der Burgt P J M and Heideman H G M 1985 J. Phys. B: At. Mol. Phys. 18 L755-61

Bukow H H, Heine G and Reinke M 1977 J. Phys. B: At. Mol. Phys. 10 2347-58

Cederquist H, Kisielinski K and Mannerik S 1983 J. Phys. B: At. Mol. Phys. 16 L479-84

Chung K T 1980 Phys. Rev. A 22 1341-5

Clark C W and Green C H 1980 Phys. Rev. A 21 1786-97

Defrance A 1980 J. Phys. B: At. Mol. Phys. 13 1229-48

Fano U 1983 Rep. Prog. Phys. 46 97-165

Fano U and Cooper J W 1965 Phys. Rev. A 138 400-2

Feshbach H 1962 Ann. Phys., NY 19 287-313

Freitas L C G, Berrington K A, Burke P G, Hibbert A, Kingston A E and Sinfailam A L 1984 J. Phys. B: At. Mol. Phys. 17 L303-9

Heddle D W O 1977 Proc. R. Soc. A 352441 -9

Heideman H G M 1980 Coherence and Correlation in Atomic Collisions ed H Kleinpoppen and J F Williams (New York: Plenum) pp 493-508

Heideman H G M, Nienhuis G and van Ittersum T 1974 J. Phys. B: At. Mol. Phys. 7 L493-5

Heideman H G M, van de Water W, Nienhuis G and Peeters P H 1976 J. Phys. B: At. Mol. Phys. 9 L523-6

Herrick D R and Kellman M E 1980 Phys. Rev. A 21 418-25

Herrick D R, Kellman M E and Poliak R D 1980 Phys. Rev. A 22 1517-35 
Hicks P J and Comer J 1975 J. Phys. B: At. Mol. Phys. 8 1866-79

Hicks P J, Cvejanović S, Comer J, Read F H and Sharp J M 1974 Vacuum 24 573-80

van Ittersum T, Heideman H G M, Nienhuis G and Prins J 1976 J. Phys. B: At. Mol. Phys. 9 1713-24

Kellman M E and Herrick D R 1980 Phys. Rev. A 22 1536-50

King G C, Read F H and Bradford R C 1975 J. Phys. B: At. Mol. Phys. 8 2210-24

Le Dourneuf M and Watanabe S 1984 10e Colloque sur la Physique des Collisions Atomiques et Electroniques, Aussois (France), Conférences invitées pp 65-9

Leisin O, Morgner H, Müller W A, Seiberle H and Stegmaier 1985 Mol. Phys. 54 1101-15

Lin C D 1974 Phys. Rev. A 10 1986-2001

1982 Phys. Rev. A 25 76-87

1983 Phys. Rev. Lett. 51 1348-51

1984 Phys. Rev. A 29 1019-33

Lipsky L, Anania R and Conneely M J 1977 At. Data Nucl. Data Tables 20 127-41

Lipsky L and Conneely M J 1976 Phys. Rev. A 14 2193-205

Madden R P and Codling K 1963 Phys. Rev. Lett. 10 516-8

— 1965 Astrophys. J. 141 364-75

Massey H S W and Burbop E H S 1969 Electronic and Ionic Impact Phenomena Vol I (Oxford: Clarendon)

Morgenstern R, Niehaus A and Thielmann U 1977 J. Phys. B: At. Mol. Phys. 10 1039-58

Nesbet R K 1976 Phys. Rev. A 14 1326-32

1978 J. Phys. B: At. Mol. Phys. 11 L21-4

Nienhuis G and Heideman H G M 1976 J. Phys. B: At. Mol. Phys. 9 2053-62

Phillips J M and Wong S F 1981 Phys. Rev. A 23 3324-7

de Raaf A J 1983 J. Phys. E: Sci. Instrum. 16 780-5

van Raan A F J, Moll P G and van Eck J 1974 J. Phys. B: At. Mol. Phys. 7 950-65

Read F H 1977 J. Phys. B: At. Mol. Phys. 10 L207-12

1983 Aust. J. Phys. 35 475-99

Read F H and Comer J 1980 Coherence and Correlation in Atomic Collisions ed H Kleinpoppen and J F Williams (New York: Plenum) pp 243-55

Roy D, Delâge A and Carette J-D 1978a J. Phys. B: At. Mol. Phys. 11 895-908

1978b J. Phys. B: At. Mol. Phys. 11 4059-66

Scott T and McDowell M R C 1975 J. Phys. B: At. Mol. Phys. 8 1851-65

1976 J. Phys. B: At. Mol. Phys. 9 2235-54

Sinanoglu O and Herrick D R 1975 J. Chem. Phys. 62 886-92

Smith A J, Hicks P J, Read F H, Cvejanović S, King G C M, Comer J and Sharp J M 1974 J. Phys. B: At. Mol. Phys. 7 L496-502

Spence D 1975 Phys. Rev. A 12 2353-60

1980 J. Phys. B: At. Mol. Phys. 13 1611-24

Watanabe S 1982 Phys. Rev. A 25 2074-98

Watanabe S, Le Dourneuf M and Pelamourgues L 1982 Colloq. Int. 334 CNRS J. Physique Suppl. 43 233-41

van de Water W, Heideman H G M and Nienhuis G 1981 J. Phys. B: At. Mol. Phys. 14 2935-50 